\title{
Disruption of Redox Homeostasis by Enzymatic Activation of a Triakylphosphine Probe in Mitochondria
}

Jade Nguyena,b, Alina Tirla and Pablo Rivera-Fuentes*a,b

aLaboratory of Organic Chemistry, ETH Zurich, 8093, Zurich, Switzerland.

${ }^{b}$ Institute of Chemical Sciences and Engineering, EPF Lausanne, 1015, Lausanne, Switzerland.

* pablo.riverafuentes@epfl.ch

ABSTRACT: Redox homeostasis is essential for cell function and its disruption is associated with cancer, metabolic and neurodegenerative diseases. Redox balance is largely regulated by the relative concentrations of reduced and oxidized glutathione. In eukaryotes, this ratio is different in each cell compartment, and disruption of the mitochondrial redox balance has been specifically linked to pathologies such as obesity and type II diabetes. Although reduced glutathione can be scavenged using electrophiles, there is a lack of probes that can produce it. In this study, we report an organellespecific reducing agent based on tributylphosphine that selectively reduces oxidized glutathione in mitochondria. This probe is activated by endogenous nitroreductases and subsequently releases tributylphosphine, as well as a fluorescent reporter, within the organelle. Confocal imaging and biological assays in human cells revealed that, counterintuitively, increased reduced glutathione induced oxidative stress through accumulation of superoxide. Transcriptomic analysis was used to establish that mitochondrial redox stress activates a cellular response orchestrated by transcription factor ATF4, which upregulates genes involved in glutathione metabolism.

\section{INTRODUCTION}

Intracellular reduction-oxidation (redox) balance is crucial for cell function and is primarily regulated by the relative concentrations of glutathione (GSH) and its oxidized, disulfide-bonded dimer (GSSG). ${ }^{1}$ Multiple physiological processes, ranging from cell signaling to protein folding depend on redox homeostasis, and therefore, several pathological conditions such as cancer, ${ }^{2}$ diabetes $^{3}$ and neurodegenerative diseases ${ }^{4}$ have been associated with 
redox imbalances. In eukaryotes, this homeostasis is controlled at the level of subcellular compartments and each organelle possesses its own redox environment. ${ }^{5}$ Being able to modulate the GSH/GSSG ratio in an organelle-specific manner would allow us to investigate the relationship between cellular stress responses and the spatial origin of the imbalance, enriching our understanding of subcellular compartmentalization of redox signaling.

Mitochondria are organelles that carry out multiple essential tasks in the cell that depend on redox modulation. Disruption of this homeostasis leads to pathologies such as insulin resistance, obesity and type II diabetes. ${ }^{6}$ Whereas the effects of oxidative stress in mitochondria have been thoroughly investigated, ${ }^{7}$ reductive stress has remained significantly underexplored. An important advance in this area was the development of enzymes that can control the ratios of oxidized and reduced nicotinamide adenine dinucleotide $\left(\mathrm{NAD}^{+} / \mathrm{NADH}\right)^{8}$ and its phosphate derivatives (NADP+/NADPH).9 Small molecules that could induce reductive stress by directly affecting the ratio of GSH/GSSG would provide additional information of how cells deal with reductive stress stemming from this essential redox buffering system.

We envisioned that the GSH/GSSG ratio could be manipulated by direct reduction of the disulfide bond in GSSG. This disulfide can be efficiently and rapidly reduced to GSH by trialkylphosphine derivatives, which have no appreciable reactivity against other amino acids.10,11 We hypothesized that we could achieve mitochondria-specific reductive stress by taking advantage of the activity of enzymes that are present only in these organelles to trigger the release of tributylphosphine from a masked precursor. Here, we report the development of such probe, its validation in live human cells, and its application to characterize the cellular response to mitochondrial reductive stress.

\section{RESULTS AND DISCUSSION}

Design, Synthesis and Enzymatic Activation. To release tributylphosphine exclusively in mitochondria, we selected endogenous nitroreductases (NTRs) as the activating agent. Although the enzymes that are responsible for this reactivity have not been fully identified in mammalian cells, they are capable of reducing nitroaromatic compounds to amines under normoxic conditions.12,13 Based on this knowledge, we developed the tributylphosphonium probe 1 with a nitro group as the enzymatically activatable trigger (Scheme 1A). Upon enzymatic reaction, the strong electron-withdrawing nitro group is converted to 
an amine, which is able to donate electron density into the $\pi$-conjugated system, cleaving the weak $\mathrm{C}-\mathrm{P}$ bond to release the reducing agent tributylphosphine $\left(\mathrm{PBu}_{3}\right)$ and fluorescent reporter 2 (Scheme 1A). Probe 1 was synthesized in three steps and intermediate $\mathbf{3}$ in two steps in overall moderate yields (Scheme 1B). Indoleninium 5 was obtained by methylation of 2, 3, 3trimethylindolenine (4) by microwave irradiation. Probes 1 and 2 were obtained by Knoevenagel condensation of 5 with 4-nitrobenzaldehyde and 4aminobenzaldehyde respectively. The phosphonium moiety was introduced by conjugate addition of n-tributyl phosphine to compound 2 to afford probe 1 in high yield.

A

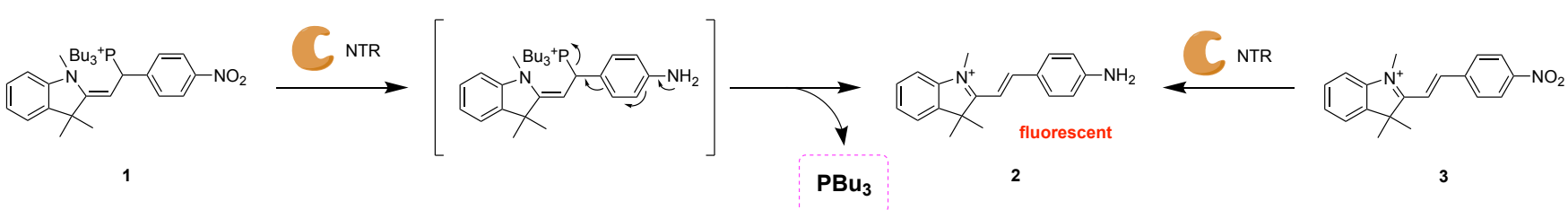

B

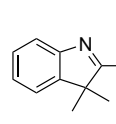

4

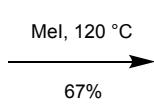

$67 \%$

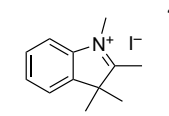

5

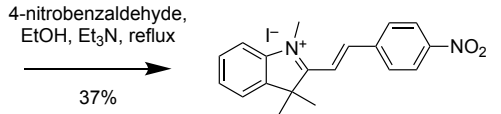

3
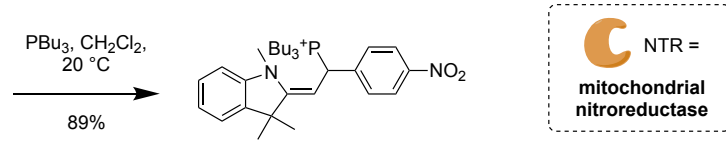

1

Scheme 1. (A) Mechanism of enzymatic activation and release of $\mathrm{PBu}_{3}$ and fluorescent reporter 2 and (B) Synthesis of probes 1 and 3 .

Probe 1 displayed no significant fluorescence emission prior to enzymatic activation because the tributylphosphonium moiety interrupts the conjugation of the $\pi$-system (Scheme $1 \mathrm{~A}$ and Table S1). Reporter dye 2, in contrast, displays strong absorption and fluorescence (Scheme $1 \mathrm{~A}$ and Table S1) and is suitable for detection by fluorescence emission (Figure S1). A purified bacterial NTR reduced the nitro group of probe 1 to produce reporter 2 with concomitant release of tributylphosphine (Figure S2). Similarly, compound 3, which is an analogue of probe 1 that lacks tributylphosphine was converted to fluorophore 2 by bacterial NTR (Figure S2).

Confocal Microscopy Experiments. Confocal microscopy experiments employing live HEK293 cells revealed that both probes 1 and 3 are activated exclusively in mitochondria (Figure S3). Probe 3 displayed more intense intracellular fluorescence than probe 1 even at lower extracellular concentrations (Figure S4). We determined the intracellular concentrations of reporter dye 2 upon activation of either probe 1 or 3 by LC-MS analysis of lysates in selected 
ion mode (LC-MS SIM). These experiments revealed that probe 3 produced more dye 2 than probe 1 at the same extracellular concentrations, possibly as a consequence of greater cellular uptake of probe 3 (Figure S5). This hypothesis is also supported by cell viability experiments (Figure S6). We estimated that probes 1 and 3 produced equivalent amounts of intracellular dye 2 at extracellular concentrations of $15 \mu \mathrm{M}$ and $5 \mu \mathrm{M}$, respectively, and used these concentrations for all subsequent cell experiments in which the effects of probe 1 and 3 are compared.

We tested whether release of tributylphosphine from probe 1 increased the intracellular concentration of GSH using a ratiometric fluorescent probe. ${ }^{14}$ When cells were treated with probe 1 for $30 \mathrm{~min}$, the GSH/GSSG ratio increased significantly compared to control probe 3 (Figure 1A). Whereas tributylphosphine can reduce GSSG rapidly, breaking disulfide bonds in proteins is greatly hindered by steric bulk. ${ }^{10}$ If probe 1 was able to break disulfide bonds from proteins, it would increase the amount of free, nucleophilic thiols in the proteome of the treated cell. Using an iodoacetamide alkyne as a general electrophilic probe and a fluorescent reporter, ${ }^{15}$ we determined that the proteomes of cells treated with probes $\mathbf{1}$, 3, or DMSO did not display significantly increased or decreased availability of free, nucleophilic thiols (Figure S7), confirming that tributylphosphine reduces the unhindered disulfide bond in GSSG preferentially. 


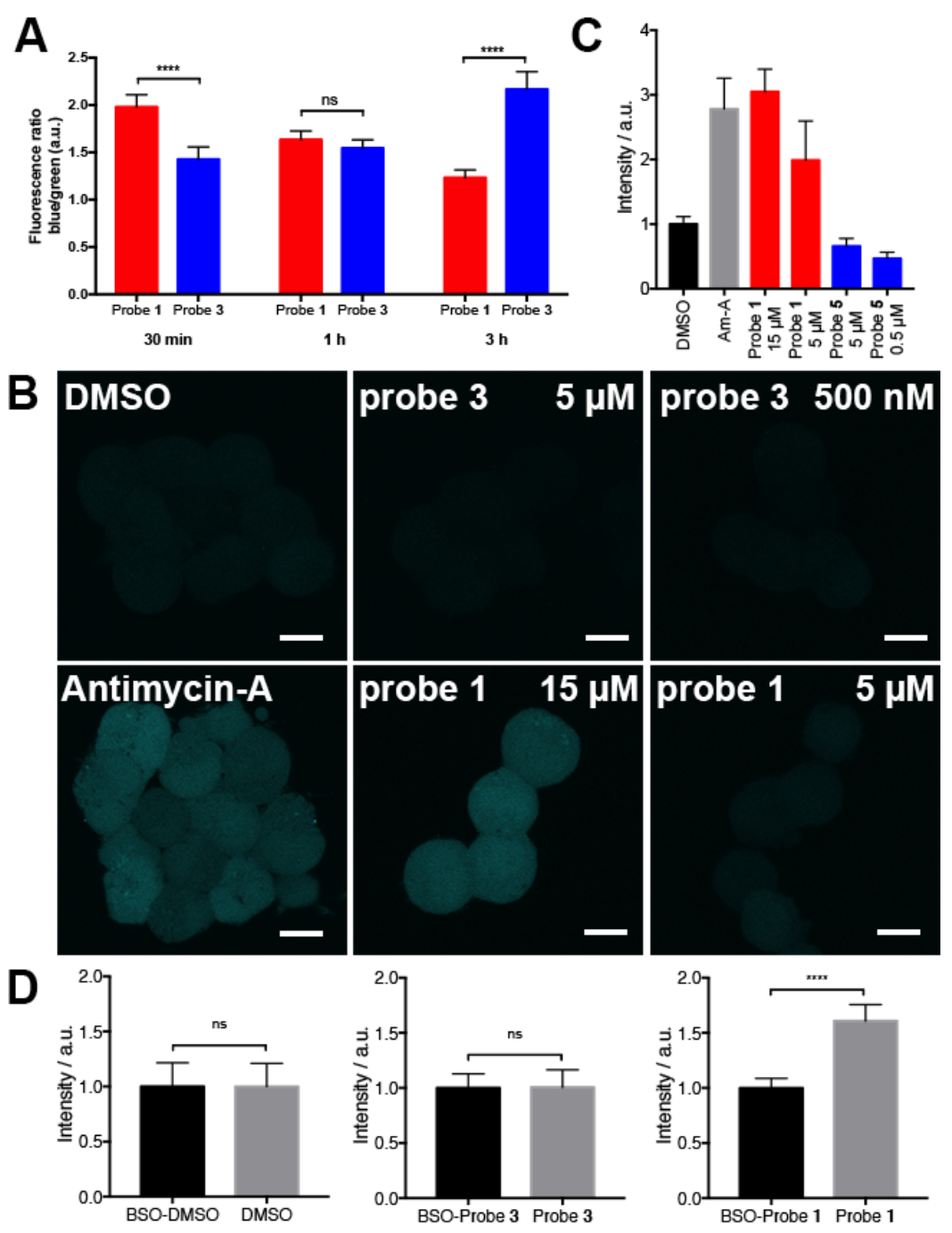

Figure 1. Effect of probe 1 on live HEK293 cells. (A) GSH/GSSG ratios. Ratios of blue/green fluorescence with a ratiometric probe $(2.5 \mu \mathrm{M}, 30 \mathrm{~min})$ for the detection of GSH (blue) and GSSG (green) after treatment with probe 1 $(15 \mu \mathrm{M})$ or $3(5 \mu \mathrm{M})$. (B) Superoxide production with $\mathrm{O}_{2}^{-}$sensor $\mathrm{HK}-\mathrm{SOX}-1$ ( $\lambda_{\mathrm{ex}}$ $=488 \mathrm{~nm})$ after treatment with DMSO (1\%, 2-3 h), Antimycin-A $(10 \mu \mathrm{M}$, $30 \mathrm{~min}$ ), probe 1 (15 $\mu \mathrm{M}$ and $5 \mu \mathrm{M}, 2-3 \mathrm{~h}$ ), or probe 3 ( $5 \mu \mathrm{M}$ and $0.5 \mu \mathrm{M}, 2-3$ h). (C) Quantification of fluorescence intensity compared to DMSO control (intensity $=1$ ) of cells treated as described in (C). (D) Quantification of $\mathrm{O}_{2}^{-}$production with $\mathrm{HK}-\mathrm{SOX}-1 \quad\left(\lambda_{\mathrm{ex}}=488 \mathrm{~nm}\right)$ after treatment with DMSO (1\%), probe $3(5 \mu \mathrm{M})$ or probe $1(15 \mu \mathrm{M})$ for $3 \mathrm{~h}$, with (intensity = 1) or without prior BSO treatment $(250 \mu \mathrm{M}, 3 \mathrm{~h})$. Means are plotted and error bars represent 95\% confidence interval. Measurements were carried out for $\mathrm{n}>50$ cells from biological triplicates. Statistical significance was assessed by unpaired, two-tailed, Mann-Whitney test. $P$ values: $* \star \star *<0.0001$ and ns $=$ not significant $>0.05$. Scale bars $=10 \mu \mathrm{m}$. 
Whereas probe 1 increased the GSH/GSSG ratio during the first $30 \mathrm{~min}$, a longer incubation time of $3 \mathrm{~h}$ led to a decrease in the GSH/GSSG ratio (Figure 1A). Previous studies have reported that an increase in GSH may lead to oxidative stress through accumulation of intracellular superoxide $\left(\mathrm{O}_{2}^{-}\right) \cdot{ }^{16}$ Using a fluorescent indicator, ${ }^{17}$ we confirmed that cells treated with probe 1 displayed significantly higher $\mathrm{O}_{2}^{-}$than cells treated with probe 3 (Figure 1B,C). Antimycin-A, an inhibitor of complex III, was used as marker for mitochondrial $\mathrm{O}_{2}^{-}$production ${ }^{17}$ and DMSO as negative control. Moreover, to test whether the increase in $\mathrm{O}_{2}^{-}$induced by probe 1 depends on the total concentration of GSH, we blocked its synthesis by inhibiting $\gamma$-glutamylcysteine synthetase using buthionine sulfoximine (BSO) (Figure 1D). ${ }^{18}$ The significant decrease in $\mathrm{O}_{2}^{-}$levels in cells depleted from GSH confirms that mitochondrial reductive stress is translated into oxidative stress by $\mathrm{O}_{2}^{-}$accumulation (Figure 1D) .

Three-dimensional confocal microscopy revealed that probe $\mathbf{1}$, but not 3, induced drastic changes in the morphology of mitochondria (Figure 2A). These changes were evaluated by quantitative image analysis (see SI for details) and revealed that mitochondria of cells treated with probe 1 displayed shorter length, greater circularity (Figure 2B), and smaller aspect ratio (Figure S8). These morphologies are reminiscent of those of depolarized mitochondria, for example upon treatment of cells with the protonophore carbonyl cyanide m-chlorophenyl hydrazone (CCCP, Figure 2A). ${ }^{19}$ CCCP is a mitochondrial uncoupler that induces membrane depolarization. ${ }^{19}$ Depolarized mitochondria leak cationic dyes, such as 2, to the cytosol, which can be observed in CCCP-treated cells (Figure 2C). In contrast, cells treated with probe 1 retained most of reporter dye 2 in mitochondria, demonstrating that release of tributylphosphine does not induce significant mitochondrial membrane depolarization (Figure 2D). Notably, CCCP also induced a much larger decrease in mitochondrial area compared to probe 1 (Figure S8), suggesting that reductive stress induced by trialkylphosphine triggers morphological changes, but not necessarily fragmentation or degradation. 

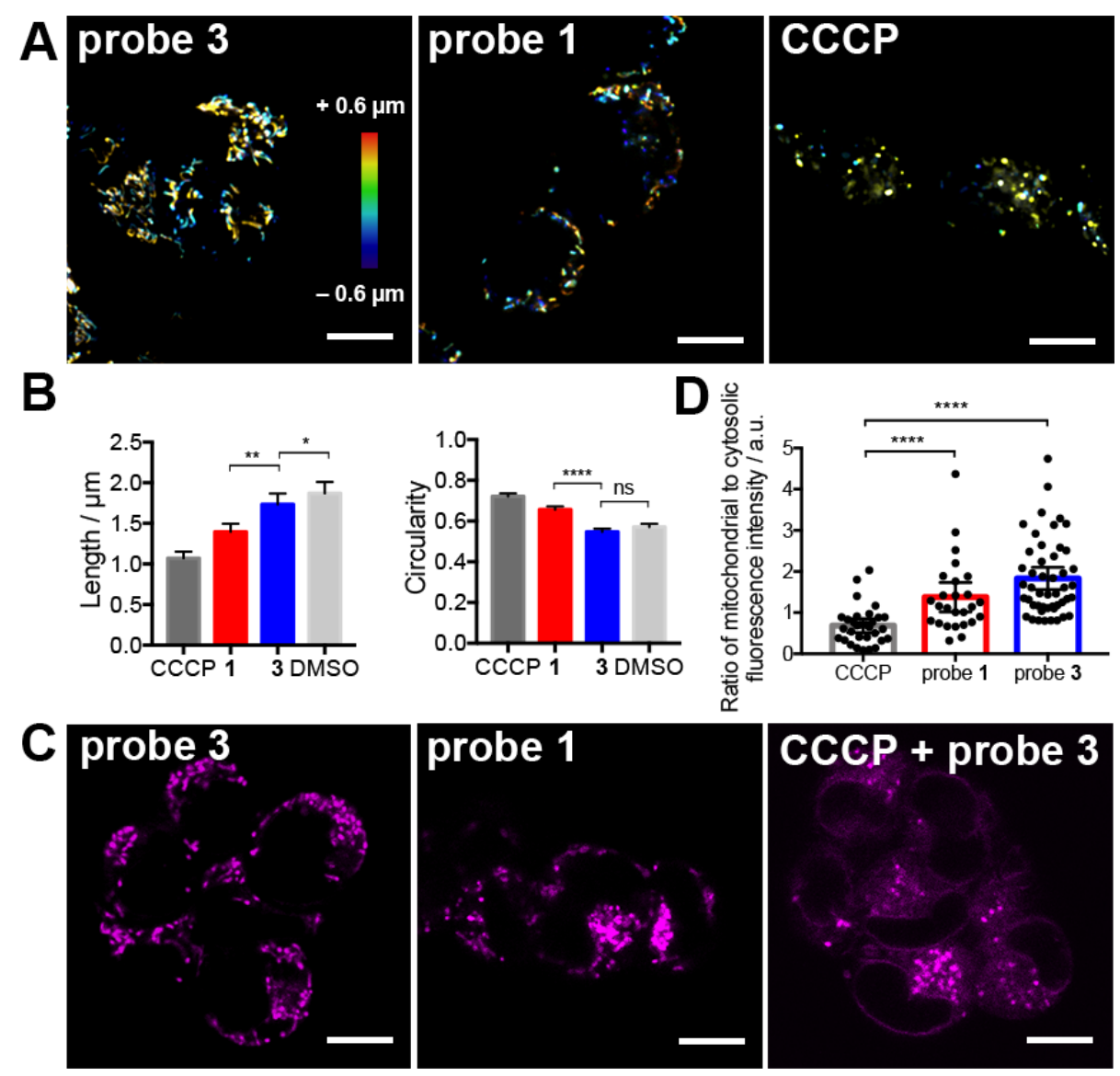

Figure 2. Effect of probe 1 on the morphology and depolarization of mitochondria in live HEK293 cells. (A) Three-dimensional confocal microscopy of

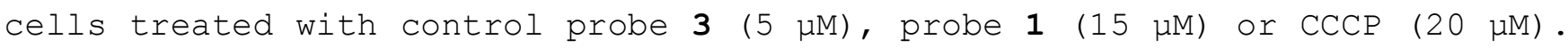
This last group of cells was stained with the dye MitoTracker Deep Red FM (MDR, $20 \mathrm{nM}$ ) for visualization. (B) Quantification of morphological parameters of cells treated with probes $\mathbf{3}$ or $\mathbf{1}$, or with CCCP or DMSO control stained with MDR. Measurements were carried out in biological triplicates and morphological data from $\mathrm{n}=1000$ mitochondria were employed for each condition. Means are plotted and error bars represent 95\% confidence intervals. (C) Mitochondrial membrane depolarization in cells incubated with probe $1(15 \mu \mathrm{M})$, probe $3(5 \mu \mathrm{M})$, or $\operatorname{CCCP}(20 \mu \mathrm{M})$ co-incubated with probe 3 $(1 \mu \mathrm{M})$ for $2 \mathrm{~h}$. (D) Quantification of the ratio of mitochondrial to cytoplasmic area-normalized fluorescence intensity. Measurements were carried out in biological triplicates and data from $n>26$ cells were employed for each condition. Means are plotted and error bars represent 95\% confidence intervals. In all cases, statistical significance was assessed by unpaired, two-tailed, Mann-Whitney test. $P$ values: $\star \star \star \star<0.0001, * \star<0.001, *<$ 0.05 and $\mathrm{ns}=$ not significant $>0.05$. Scale bars $=10 \mu \mathrm{m}$. 
Severely damaged mitochondria undergo degradation and recycling through a selective autophagic process known as mitophagy. ${ }^{20}$ This process involves recruitment of the ubiquitin ligase parkin to the membrane of depolarized mitochondria, ${ }^{21}$ ubiquitination, engulfment by the autophagosome, and degradation of the organelle (Figure 3A). ${ }^{22}$ To assess whether reductive stress induced by probe 1 triggers mitophagy, we transfected cells with either parkin or the autophagosomal marker LC3 fused to the bright fluorescent protein mTurquoise2 (Figure S9 and Table S2). ${ }^{23}$ No recruitment of parkin to the mitochondrial membrane was observed in cells treated with probe 1 . In contrast, cells treated with CCCP recruited parkin efficiently (Figure 3B). We also analyzed the number of LC3 punctae (autophagosomes) that co-localized with mitochondria in cells under various treatments using a custom-made algorithm for image analysis (Figure $3 \mathrm{C}$ and SI). We did not find a significant difference between cells treated with probe 1 or DMSO, whereas cells treated with rapamycin, an inductor of autophagy, displayed more co-localized punctae (Figure 3C). ${ }^{24}$ These observations indicate that mitochondrial reductive stress induced by tributylphosphine does not trigger mitophagy. 

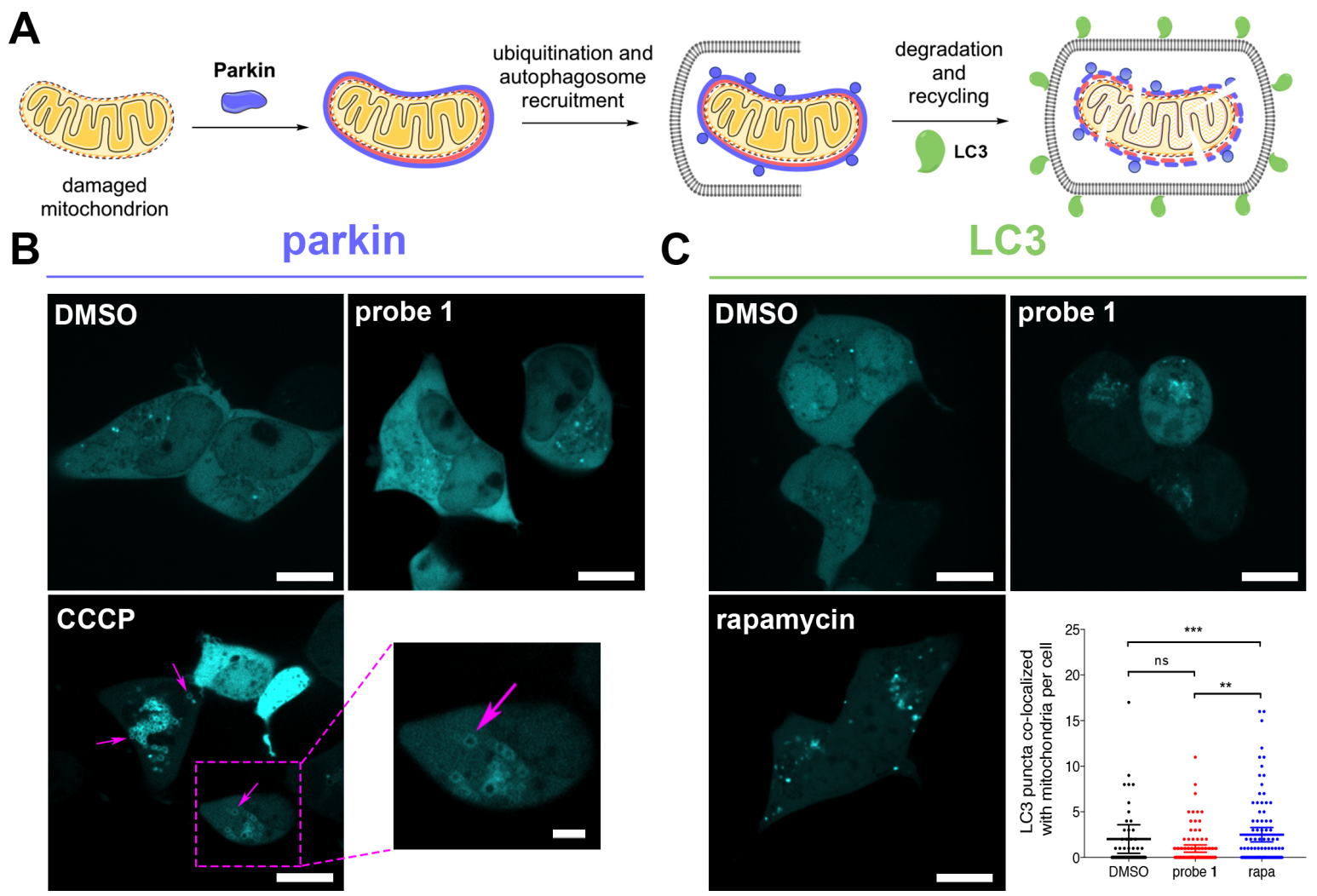

Figure 3. Tributylphosphine-induced reductive stress does not trigger mitophagy in live HEK293 cells. (A) Mechanism of parkin-mediated mitophagy. (B) Confocal imaging of cells expressing mTurquoise2-parkin and treated with probe $1(15 \mu \mathrm{M})$, DMSO, or CCCP $(20 \mu \mathrm{M})$. Arrows indicate examples of parkin recruited to damaged mitochondria. (C) Confocal imaging of cells expressing mTurquoise2-LC3 and treated with probe 1 (15 $\mu \mathrm{M})$, DMSO, or rapamycin (rapa, $1 \mathrm{\mu M})$. Quantification of number of LC3 punctae that co-localized with mitochondria per cell under the conditions mentioned above. Means are plotted and error bars represent 95\% confidence interval. Measurements were carried out for $n>50$ cells from biological triplicates. Statistical significance

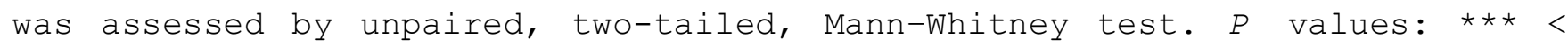
$0.001 ; * *<0.01$ and $\mathrm{ns}=$ not significant $>0.05$. Scale bars $=10 \mu \mathrm{m}$ and $2 \mu \mathrm{m}$ (inset in panel B).

Transcriptomic Analysis. Global mRNA sequencing and differential gene expression analysis revealed that cells treated with probe 1 displayed significant upregulation of several genes, particularly $\gamma$-glutamyl cyclotransferase (CHAC1), compared to DMSO-treated cells (Figure 4A). On the other hand, probe 3 induced the upregulation of only very few genes compared to DMSO treatment (Figure 4B) and the products of these genes had very different 
cellular and molecular functions compared to probe 1 (Figure S10). This comparison confirms that redox stress induced by tributylphosphine is responsible for the upregulation of genes observed with probe 1 . CHACl regulates redox homeostasis through degradation of GSH,25 suggesting that the cell responds to reductive stress by promoting the depletion of excess GSH produced by tributylphosphine. CHACl is a component of the PERK branch of the unfolded protein response in the endoplasmic reticulum (UPR $\left.{ }^{E R}\right) .{ }^{26}$ In this case, however, upregulation of CHACl is not mediated by the UPRER, because probe 1 did not trigger upregulation of the ER stress marker BiP (Figure 4C), which is involved in all three branches of the UPR ${ }^{\mathrm{ER}}$ pathway. ${ }^{26}$ In addition, other classical activators of the UPR ${ }^{\mathrm{ER}}$ such as ATF6 or IRE1 $\alpha$, or target genes of this response, such as chaperone GRP94, phosphatase GADD34, or disulfide isomerase PDIA6 were not affected by either probe $\mathbf{1}$ or $\mathbf{3}$ (Figure 4C). These results indicate that upregulation of CHACl is not triggered by the UPR, further confirming the organelle selectivity of probe 1 .

The UPR in mitochondria (UPR ${ }^{\mathrm{mt}}$ ) can be activated by employing the mitochondria-specific HSP90 inhibitor gamitrinib-triphenylphosphonium (GTPP), which leads to upregulation of genes HSPD1 and HSPE1.27 Activation of tributylphosphine in mitochondria did not alter transcription of either of these genes (Figure 4C). Additionally, GTPP induces parkin-mediated mitophagy, 28 whereas probe 1 does not (Figure 3B). These results strongly suggest that reductive stress does not trigger the $U P R^{\mathrm{mt}}$, or at least not in the way that chaperone inhibitors such as GTPP do.

Our mRNA sequencing results revealed that transcription factors ATF4, ATF3 and CHOP were upregulated by probe 1 (Figure 4D). These observations are consistent with a recent multi-omics characterization of the stress response to inhibitors of mitochondrial import, translation, membrane potential, or oxidative phosphorylation. ${ }^{29}$ This study demonstrated that these mitochondrial insults triggered the integrated stress response (ISR), which is regulated by ATF4 and activates CHAC1.29 This study, however, also reported significant upregulation of genes involved in amino acid metabolism, such as asparagine synthetase (ASNS) and phosphoserine phosphatase (PSPH). ${ }^{29}$ These genes were only mildly upregulated by reductive stress induced by probe $\mathbf{1}$ (Figure 4D). It is therefore clear that even though tributylphosphine shares some common features with various mitochondrial inhibitors, for example increased production of $\mathrm{O}_{2}^{-}$(Figure 1B), ${ }^{16}$ the cellular response that it elic- 
its is unique and specifically addresses the imbalance in GSH/GSSG through CHAC1 upregulation (Figure 4E).

A

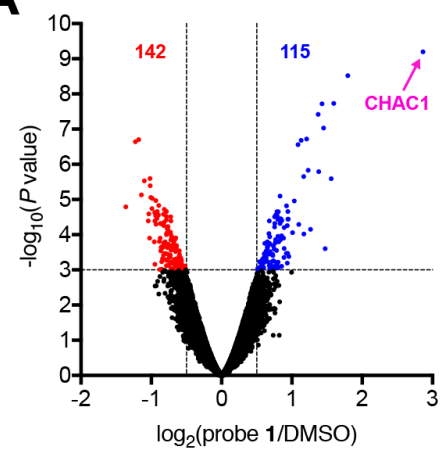

B

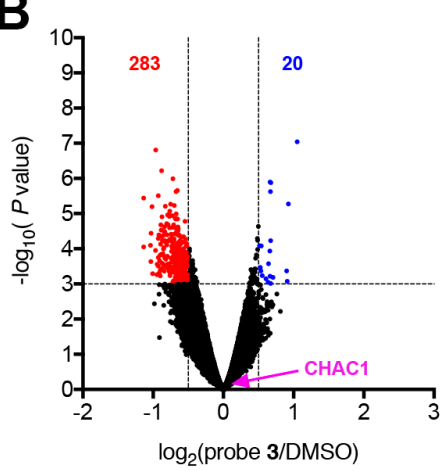

D ISRgenes

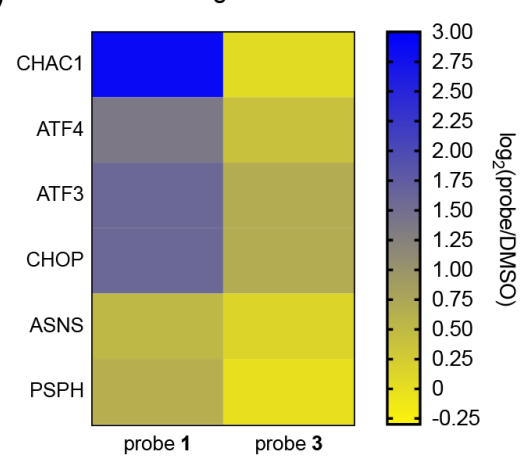

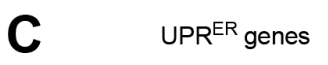

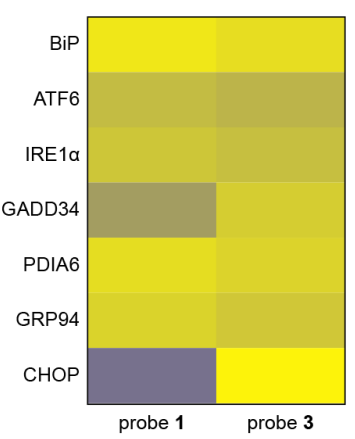

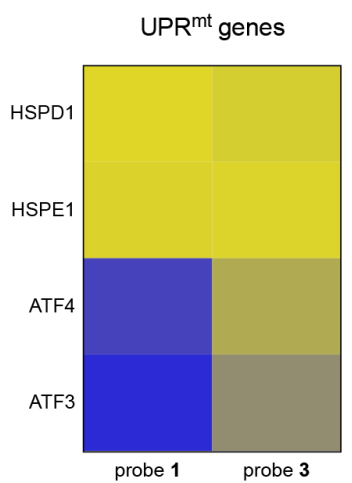

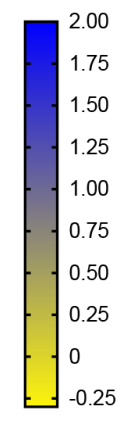

E

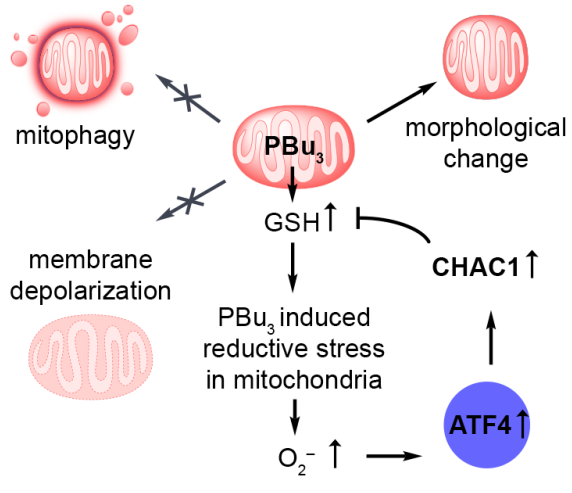

Figure 4. Transcriptional response to mitochondrial reductive stress induced by tributylphosphine. (A,B) Volcano plots of differential gene expression in cells treated with probe 1 (15 $\mu \mathrm{M})$ (A) or with probe 3 (5 $\mu \mathrm{M})$ (B) and compared against cells treated with DMSO. Genes and number of genes that are significantly upregulated are depicted in blue, those that are significantly downregulated depicted in red, using thresholds of $P<0.001$ and $\log _{2}(\mathrm{probe} / \mathrm{DMSO})>0.5$ or $<-0.5$ obtained from biological triplicates $(\mathrm{n}=$ 3). (C,D) Differential regulation of genes involved in the UPR or UPR ${ }^{\mathrm{mt}}$ (C)

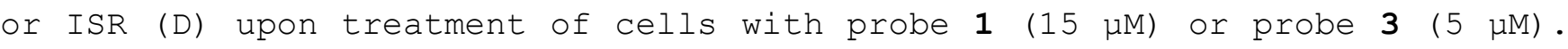
(E) Proposed mechanism of action of tributylphosphine in mitochondria.

Summary and Conclusions. In summary, selective release of tributylphosphine in mitochondria reduced GSSG to increase the ratio of GSH/GSSG without appreciable reduction of disulfide bonds in proteins. Reductive stress is eventually transformed into oxidative stress through the production of $\mathrm{O}_{2}^{-}$. This redox imbalance does not depolarize mitochondria or activate stress responses like the UPR ${ }^{E R}$, UPR ${ }^{\mathrm{mt}}$, or mitophagy. Instead, it activates the ATF4ATF3-CHOP cascade, which upregulates the CHAC1 gene (Figure 4E) . 
Our observations are consistent with reports of mitochondrial oxidation triggered by glutathione-dependent reductive stress ${ }^{16}$ and activation of ATF4 by $\mathrm{O}_{2}^{-} \cdot{ }^{18}$ We hypothesize that the increased levels of GSH in mitochondria can lead to glutathionylation of superoxide dismutase (SOD1), preventing the disproportionation of $\mathrm{O}_{2}{ }^{-},{ }^{30}$ but this hypothesis remains to be proved.

These results indicate that trialkylphosphines, a broad family of compounds that have been largely neglected in chemical biology, can expand the chemical space of small molecules that are used to modulate redox biology, with potential impact in the development of new therapies. For example, CHAC1 upregulation has been reported to deplete GSH levels in triple-negative breast cancer cells, making them more susceptible to necroptosis and ferroptosis during cystine starvation. ${ }^{1}$ In this work, we have demonstrated that even though trialkylphosphines are highly reducing and often water-insoluble compounds, they can be transformed into chemical probes for biological use by developing strategies to tune their reactivity, mask their reducing power, target them to specific organelles, and release them selectively.

\section{MATERIALS AND METHODS}

General methods. All reagents were purchased from commercial sources and used without further purification. Solvents were obtained from Sigma-Aldrich and used as received. NMR spectra were acquired on a Bruker AV300 or Bruker 400 instruments. ${ }^{1} \mathrm{H}$ NMR chemical shifts are reported in ppm relative to SiMe4 $(\delta=0)$ and were referenced internally with respect to residual protons in the solvent $\left(\delta=1.94\right.$ for $\mathrm{CD}_{3} \mathrm{CN}, \delta=3.31$ for $\mathrm{CD}_{3} \mathrm{OD}$ and $\delta=2.50$ for DMSO-d6). Coupling constants are reported in $\mathrm{Hz} \cdot{ }^{13} \mathrm{C}$ NMR chemical shifts are reported in ppm relative to $\mathrm{SiMe}_{4}(\delta=0)$ and were referenced internally with respect to solvent signal $\left(\delta=118.3\right.$ for $\mathrm{CD}_{3} \mathrm{CN}, \delta=49.0$ for $\mathrm{CD}_{3} \mathrm{OD}$ and $\delta$ $=39.5$ for DMSO-d6). Peak assignments are based on calculated chemical shifts, multiplicity and 2D experiments. IUPAC names of all compounds are provided and were determined using CS ChemBioDrawUltra 16. High-resolution mass spectra (HRMS) were recorded by staff at the Molecular and Bioanalytical (MoBiAS) center (ETH Zurich) employing a Bruker maXis-ESI-Qq-TOF-MS (ESI). Description of syntheses, spectroscopic characterization, and NMR spectra are reported in the supporting information.

Optical spectroscopy. Stock solutions in DMSO were prepared at concentrations of 1-15 mM, stored at $-20{ }^{\circ} \mathrm{C}$ and thawed immediately before each exper- 
iment. Spectroscopic measurements were conducted in phosphate-buffered saline (PBS) at $\mathrm{pH}=7.4$. UV-Visible spectra were obtained employing a Cary 500 Scan spectrometer using quartz cuvettes from Thorlabs (10 mm path length). Fluorescence spectra were acquired using a Fluorolog 3 fluorimeter (Horiba Jobin-Yvon) at the indicated temperature. Extinction coefficients were determined by linear fit of 4 different concentrations of the compound in PBS. Absolute fluorescence quantum yields were determined in PBS by means of an integrating sphere (Horiba Jobin-Yvon). All spectroscopic experiments were carried out in triplicate.

Enzyme kinetics. Michaelis-Menten kinetics experiments were performed by monitoring the formation of $\mathbf{2}$ at different concentrations of substrate 1 or $3(2-60 \mu \mathrm{M})$, constant NTR (2 $\left.\mu \mathrm{g} \mathrm{mL}^{-1}\right)$ and NADH $(500 \mu \mathrm{M})$ concentrations in PBS at $37{ }^{\circ} \mathrm{C}$. The initial velocities of enzymatic cleavage were obtained by monitoring the absorbance of 2 in the first $10 \mathrm{~min}$ of the enzymatic activation. Because the absorbance of 2 is directly proportional to its concentration, the absorbance could be expressed in terms of concentration and the initial velocities $\left(V_{0}\right)$ at different concentrations of probe 1 or 3 could be calculated. The velocities were plotted against the initial concentrations of 1 or 3. The data were fit using the Michaelis-Menten equation in Prism 7 (GraphPad) to give the Michaelis-Menten constant ( $\left.K_{m}\right)$ and turnover number $\left(k_{\text {cat }}\right)$.

Cloning. All plasmids were cloned by Gibson assembly. ${ }^{32}$ DNA encoding for mTurquoise2 was amplified from plasmid pmTurquoise2-ER (Addgene \#36204) to generate an insert. Plasmids containing the gene of interest fused to another fluorescent protein were used to generate the backbone. These plasmids were CFP-Parkin (Addgene \#47560) and pEGFP-LC3 (Addgene \#24920). Primers for amplification (minimum 15 overlapping base pairs) were generated using SnapGene ${ }^{\circledR}$ and modified manually to minimize hairpins, homodimers, and repeating motifs. The designed primers (Table S2) were supplied by Mycrosynth AG (Switzerland). The amplified DNA fragments were generated by PCR using Phusion High-Fidelity PCR Master Mix with HF from New England Biolabs (NEB). The fragments were analyzed by gel electrophoresis and purified using QIAquick PCR purification kit (Qiagen) according to the manufacturer's instructions. The Gibson assembly reaction was performed using Gibson Assembly Master Mix (NEB). NEB DH5 $\alpha$ competent E. coli cells were transformed with the assembly product according to the manufacturer's protocol and streaked onto lysogeny broth (LB) agar plates containing kanamycin (50 $\mu \mathrm{g} \mathrm{mL}^{-1}$ ). After incubation at $37{ }^{\circ} \mathrm{C}$ for $24 \mathrm{~h}$, single colonies were selected and grown in LB 
liquid medium containing $50 \mu \mathrm{g} \mathrm{mL} \mathrm{m}^{-1}$ kanamycin at $37^{\circ} \mathrm{C}$ for $16 \mathrm{~h}$. Plasmid DNA was extracted and purified using QIAprep spin miniprep kit (Qiagen) according to the manufacturer's instructions and sequenced by Mycrosynth AG (Switzerland). Plasmids are available from Addgene as pLAMP1-mTurquoise2 (pmTLyso, \#110948), pmTurquoise2-Parkin (pmT-Parkin, \#110945) and pmTurquoise2LC3 (pmT-LC3, \#110947).

Cell culture. HEK293 cells were grown in Dulbecco's Modified Eagle Medium (DMEM) supplemented with fetal bovine serum (FBS, 10\%) and penicillinstreptomycin (1\%) at $37{ }^{\circ} \mathrm{C}$ in $5 \% \mathrm{CO}_{2}$ environment. The cells were split after 90\% confluence was reached. For microscopy experiments, 20'000 cells per well were seeded onto 8 -well plates $(0.5 \mathrm{~mL}$, Nunc Lab-Tek II or $0.3 \mathrm{~mL}$, Ibidi chambered cover glass) 2-4 days prior to imaging. The probes were incubated in growth medium for the indicated time. Before imaging, the growth medium was removed, and the cells were washed with PBS and imaged in imaging medium (FluoroBrite DMEM) .

Cell viability assays. Cell viability assays. HEK293 cells were grown in DMEM supplemented with FBS (10\%) and penicillin-streptomycin (1\%) at $37{ }^{\circ} \mathrm{C}$ in 5\% $\mathrm{CO}_{2}$ environment. The cytotoxicity of the probes was determined by methylthiazolyldiphenyl-tetrazolium bromide (MTT) colorimetric assays. MTT is reduced to the purple tetrazolium salt formazan in living cells. 9'000 cells were seeded onto each well of a 96-well plate and grown for $24 \mathrm{~h}$ at $37{ }^{\circ} \mathrm{C}$, 5\% $\mathrm{CO}_{2}$. The compounds were incubated for $48 \mathrm{~h}$ at different concentrations ranging from $100 \mathrm{nM}$ to $100 \mathrm{\mu M}$. Stock solutions of the compounds were prepared in DMSO and incubated in growth medium with a final DMSO concentration of $0.5 \%$. Positive control with $0.5 \%$ DMSO in growth medium was taken as the reference for $100 \%$ cell viability. After $48 \mathrm{~h}$ of incubation, the cells were treated with 10\% MTT solution ( $5 \mathrm{mg} \mathrm{mL}^{-1}$ in FluoroBrite DMEM) and incubated for $3 \mathrm{~h}$. The medium was then removed carefully and 2-propanol (100 $\mu \mathrm{L}$ per well) was added to dissolve the formazan. The plates were shaken at $450 \mathrm{rpm}$ for $30 \mathrm{~min}$ and the absorbance was measured at $550 \mathrm{~nm}$, using a plate reader (SPARK 10M, TECAN Group AG). Biological triplicates, each measured three times $(n=9)$, for each compound were recorded for each concentration and IC $_{50}$ values were determined using Prism 7 (Graph Pad) fit with a four parameters logistic regression.

Confocal fluorescence microscopy. Imaging was performed with a spinning disk confocal (Nikon) or a confocal microscope (Olympus). The Nikon Eclipse T1 spinning disk light microscope is equipped with a Yokogawa confocal scanner unit CSU-W1-T2 and two sCMOS (Orca Flash 4.0 V2) or EMCCD (iXon 888) camer- 
as. Light sources: Diode-pumped solid-state (DPSS) $405 \mathrm{~nm}$ (120 mW, 20-40\% laser power, 1-2 s exposure time), DPPS $445 \mathrm{~nm}(100 \mathrm{~mW}, 20-40 \%$, 1-2 s), diode $561 \mathrm{~nm}(200 \mathrm{~mW}, 20-40 \%, 1-2 \mathrm{~s})$ and $640 \mathrm{~nm}(150 \mathrm{~mW}, 40 \%, 2 \mathrm{~s})$. All images were collected using an oil-immersion 100x CFI Apo TIRF (NA = 1.49) objective. The following emission filters were used: ET450/50, ET470/24, ET 630/75, and ET700/75. The microscope was operated using VisiVIEW (Metamorph) software, the image analysis was carried out using Fiji (ImageJ $1.5 \mathrm{~d}, \mathrm{NIH}$ ) or ICY (GPLV3, Institute Pasteur). The Olympus FluoView 3000 IX83 laser scanning confocal microscope is fully-motorized and automated inverted. It is equipped with 4 GaAsP photomultiplier tubes (PMT, spectral detection option) and 1 transmission PMT. Light sources: LED based (CoolLED pE300white) unit for conventional epi-illumination for visual observation. All images were collected using a silicon-immersion 60x Silicon UPlanSApo $(\mathrm{NA}=1.3)$ objective. The microscope was operated using FV3000 system (FV31S-SW, Cellsens) software. The image analysis was carried out using Fiji (ImageJ 1.5d, NIH). To determine intracellular fluorescence intensity, the cell body was selected as the region of interest (ROI) and the integrated intensity within the ROI was measured. An additional ROI of the same size and shape was used to obtain the integrated intensity of the background (region with no cell). The background intensity was subtracted from that of the cellcontaining ROI. The analyses were plotted using Prism 7 (GraphPad) .

Co-localization experiments. HEK293 cells were transfected $24-48$ h before imaging using plasmids obtained from Addgene (abbreviation, \#plasmid): pmTurquoise2-ER (pmT-ER, \#36204), pmTurquoise2-Mito (pmT-Mito, \#36208), pmTurquoise2-Golgi (pmT-Golgi, \#36205) 33 $^{33}$ or employing our own assembled plasmids (available from Addgene): pLAMP1-mTurquoise2 (pmT-Lyso, \#110948), pmTurquoise2-Parkin (pmT-Parkin, \#110945) and pmTurquoise2-LC3 (pmT-LC3, \#110947). Cells were transfected employing the jetPRIME kit according to the instructions from the manufacturer (Polyplus) and the medium was replaced with fresh growth medium after 4-5 h. For nucleus co-localization, cells were stained with Hoechst 33342 (5 $\mu \mathrm{M})$ for at least 30 min prior to imaging. For small molecule mitochondria co-localization, cells were incubated with MitoTracker ${ }^{\mathrm{TM}}$ Deep Red FM (MDR, Thermo Fisher, 20 nM) for 1 h prior to incubation with the probes. Compound 1 or 5 were added $1-3 \mathrm{~h}$ prior to imaging. Fluorescent emission of the activated probes (561 nm, 2 s exposure time, 40\% laser power), of the pmTurquoise2-tagged protein (445 nm, 2 s, 40\%), Hoechst (405 nm, $1 \mathrm{~s}, 40 \%)$ and MDR (640 nm, 2 s, 40\%) were recorded. Pearson's coefficients were calculated by selecting a transfected cell as ROI and running 
the co-localization studio plugin contained in the imaging program ICY (GPLv3, Institute Pasteur).

LC-MS analysis. Determination of intracellular concentrations of dye 2 by LC-MS analysis of cell lysates. This procedure was adapted from a published method. ${ }^{34}$ HEK293 cells were grown in DMEM supplemented with FBS (10\%) and penicillin-streptomycin (1\%) at $37{ }^{\circ} \mathrm{C}$ in $5 \% \mathrm{CO}_{2}$ environment. They were seeded in T75 flasks $\left(75 \mathrm{~cm}^{2}\right)$ and grown to $90 \%$ confluency. The cells were incubated with the compounds for the indicated times and concentrations. The cells were trypsinized, counted and pelleted. The medium was removed gently without disturbing the cell pellet. The cells were washed once with $1 \mathrm{~mL}$ of growth medium to avoid reducing the estimated concentration due to rapid diffusion of small molecules. The medium was removed and $200 \mu L$ of water/methanol (40:60) were added to the cell pellet, which was re-suspended by dragging the tube on a rough surface (no mixing with pipet). The cells were lysed by freeze-thaw cycles alternating $3 \mathrm{~min}$ in liquid nitrogen with thawing in water bath (room temperature) for 10 cycles. The lysate was vortexed and centrifuged for $30 \mathrm{~min}$ at $4{ }^{\circ} \mathrm{C}$. The supernatant was transferred to a new tube and centrifuged for $30 \mathrm{~min}$ at $4{ }^{\circ} \mathrm{C}$. The supernatant was transferred to a 3K MWCO Amicon tube and centrifuged for $30 \mathrm{~min}$ at $4{ }^{\circ} \mathrm{C}$ to remove molecules with a molecular weight over $3 \mathrm{kDa}$. The flow-through was stored at $-20{ }^{\circ} \mathrm{C}$ or used directly and pipetted into an LC-MS insert. Control lysates (no compound incubation) were combined and aliquoted $(5 \times 20 \mu L)$. The samples were analyzed by LC-MS using the selected ion monitoring (SIM) mode for the molecular weight of reporter $2(\mathrm{~m} / \mathrm{z}=277.5)$ and monitoring at $501 \mathrm{~nm}$, near the absorption maximum. A calibration curve was built by addition of known amounts of probe 2 to control cell lysate to generate solutions of known concentrations of $\mathbf{2}$. The intracellular concentration of dye 2 resulting from activation of probe 1 or 3 was calculated based on the calibration curve, using the absorbance peaks area, total volume of lysate and total number of cells. From each sample, $2 \mu \mathrm{L}$ were injected on a Waters Acquity Hclass UPLC connected to a Water SQ Detector 2. The prepared sample was separated on a Waters $\mathrm{BEH}$ C18 $1.7 \mu \mathrm{m}$ column with an acetonitrile $(\mathrm{ACN}) /$ water/0.1\% formic acid (FA) solvent system. Starting conditions were kept for 2 minutes at 99.9\% water in $0.1 \%$ FA. In 2 minutes, the gradient was linearly ramped to $98 \% \mathrm{ACN}$ in $0.1 \% \mathrm{FA}$. This condition was kept for $5 \mathrm{~min}$ before returning to starting conditions. The single quadrupole detector scanned alternatively between full scan $(120-650 \mathrm{~m} / \mathrm{z})$ and SIM mode, monitor- 
ing $277.5 \mathrm{~m} / \mathrm{z}$ with a dwell time of $0.125 \mathrm{~s}$. Cone voltage was kept constant at $35 \mathrm{~V}$.

GSH to GSSG ratios in live cells. Live HEK293 cells were seeded on Ibidi plates 2 days prior imaging. The cells were incubated with the corresponding compounds at the indicated concentrations and incubation times. The medium was removed, the cells were washed with PBS and incubated with GSH sensor (2.5 $\mu \mathrm{M}, 30 \mathrm{~min})$. The medium was removed, the cells were washed with PBS and imaged in FluoroBrite DMEM. The image analysis was carried out using Fiji (ImageJ $1.5 \mathrm{~d}, \mathrm{NIH}$ ). To determine intracellular fluorescence intensity, the cell body was selected as ROI and the integrated intensity within the ROI was measured in the blue channel. An additional ROI of the same size and shape was used to obtain the integrated intensity of the background (region with no cell). The background intensity was subtracted from that of the cell-containing ROI. The same ROI and background were selected in the green channel. The ratio of the background corrected blue/green channel was then calculated. The analyses were plotted using Prism 7 (GraphPad).

Labeling of free nucleophilic thiols in proteomes of treated cells. Cell lysates: HEK293 cells were grown in DMEM supplemented with FBS (10\%) and penicillin-streptomycin (1\%) at $37{ }^{\circ} \mathrm{C}$ in $5 \% \mathrm{CO}_{2}$ environment. The cells were seeded in a T25 flask and after 90\% confluence was reached, the cells were treated with the indicated compounds in growth medium: probe 1 (15 $\mu \mathrm{M}, 3 \mathrm{~h})$, probe $3(5 \mu \mathrm{M}, 3 \mathrm{~h})$, DMSO $(0.5 \% \mathrm{v} / \mathrm{v}, 3 \mathrm{~h})$, then washed with PBS. The cells were trypsinized and centrifuged, and the medium was removed. The cells were re-suspended in cold PBS, centrifuged and PBS was removed carefully without disturbing the cell pellet. The cells were re-suspended in $200 \mu \mathrm{L}$ of water/methanol (40:60) containing protease inhibitors and iodoacetamide alkyne (IAA) $(100 \mu \mathrm{M})$ except for the alkyne-free sample. The cells were lysed by freeze-thaw cycles alternating $3 \mathrm{~min}$ in liquid nitrogen with thawing in water bath (room temperature) for 10 cycles. The lysate was vortexed and centrifuged for $30 \mathrm{~min}$ at $4{ }^{\circ} \mathrm{C}\left(21^{\prime} 000 \times \mathrm{g}\right)$. The supernatant was transferred to a new tube and centrifuged for $30 \mathrm{~min}$ at $4{ }^{\circ} \mathrm{C}\left(21^{\prime} 000 \times \mathrm{g}\right)$. The supernatant was transferred to a 3K MWCO Amicon tube and centrifuged for $30 \mathrm{~min}$ at $4{ }^{\circ} \mathrm{C}$ $(14,000 \times 9)$ to separate the proteins from molecules with a molecular weight under $3 \mathrm{kDa}$. The flow-through was discarded and the protein concentration was determined by BCA assay, the samples were stored at $-20{ }^{\circ} \mathrm{C}$ for later use. 
Click-reaction: The protein concentrates were thawed on ice. The click-mix solution containing $1.25 \mathrm{mM}$ CuSO4, $2.5 \mathrm{mM}$ bathophenanthroline disulfonic acid (BPSA) and $20 \mathrm{mM}$ sodium ascorbate was prepared. For each reaction, the following was added to an Eppendorf: $45 \mu \mathrm{L}$ of protein concentrate (1-5 $\mathrm{mg} / \mathrm{mL}), 1 \mu \mathrm{L}$ of $1 \mathrm{mM}$ azide fluorophore, $4 \mu \mathrm{L}$ of the click-mix and the tube was gently flicked to avoid air bubbles. The reaction was incubated for $1 \mathrm{~h}$ at $4{ }^{\circ} \mathrm{C}$ and allowed to proceed protected from light.

Sample preparation for gel analysis: $150 \mu \mathrm{L}$ of methanol were added to $50 \mu \mathrm{L}$ of reaction mixture, vortexed briefly. $40 \mu \mathrm{L}$ of chloroform were added, vortexed briefly. The Eppendorf was centrifuged for $5 \mathrm{~min}$ at $13^{\prime} 000 \times \mathrm{g}$ and the upper aqueous layer was removed. $120 \mu \mathrm{L}$ of methanol were added and vortexed briefly. The solution was centrifuged for 5 min at $13^{\prime} 000 \times 9$ to pellet proteins and the supernatant was removed. $120 \mu \mathrm{L}$ of methanol were added, vortexed briefly, centrifuged for $5 \mathrm{~min}$ at $13^{\prime} 000 \times \mathrm{g}$ to pellet proteins, and the supernatant was removed. The protein pellet was allowed to air-dry for at least $15 \mathrm{~min}$. The samples were capped and stored at $-20{ }^{\circ} \mathrm{C}$ until ready for use.

Gel analysis: The proteins were loaded on 10-well gels from BioRad (4-20\% Mini-PROTEAN@ TGX Precast Protein Gels, 10-well, $30 \mu \mathrm{L}$ ) and ran at $200 \mathrm{~V}$ for $40 \mathrm{~min}$ in TGS running buffer. The fluorescence was read at $560 \mathrm{~nm}$ with a ChemiDoC ${ }^{\mathrm{TM}}$ MP Imaging Systems (BioRad) and the band intensities were measured using Fiji software.

Superoxide production and BSO experiment. Live HEK293 cells were seeded on Ibidi plates 2 days prior imaging. The cells were incubated with BSO for 3 $\mathrm{h}$. Then the cells were washed with PBS and co-incubated with BSO (250 $\mu \mathrm{M})$ and the corresponding compounds at the indicated concentrations for another $3 \mathrm{~h}$. The medium was removed, the cells were washed with PBS and incubated with superoxide sensor HKSOX-1 (10 $\mu \mathrm{M})$ for $30 \mathrm{~min}$. The medium was removed, the cells were washed with PBS and imaged in FluoroBrite DMEM. The image analysis was carried out using Fiji (ImageJ $1.5 \mathrm{~d}$, NIH). To determine intracellular fluorescence intensity, the cell body was selected as ROI and the integrated intensity within the ROI was measured in the green channel. An additional ROI of the same size and shape was used to obtain the integrated intensity of the background (region with no cell). The background intensity was subtracted from that of the cell-containing ROI. The analyses were plotted using Prism 7 (GraphPad). 


\section{Quantitative image analysis of cell morphologies and mitochondrial/cytosolic}

ratios. Automatic identification of mitochondria, vesicles and cytosolic space from micrographs was carried out employing homemade macros running on the Fiji platform. The code and a tutorial-like description of how they were used are provided in the Supporting Information.

Total RNA isolation. HEK293 cells were grown in DMEM supplemented with FBS (10\%) and penicillin-streptomycin (1\%) at $37{ }^{\circ} \mathrm{C}$ in $5 \% \mathrm{CO}_{2}$ environment. The cells were seeded in a T75 flask and after 90\% confluence was reached, the cells were treated with the indicated compounds for $3 \mathrm{~h}$ : DMSO (1\% V/v), probe $1(15 \mu \mathrm{M})$, or probe $3(5 \mu \mathrm{M})$. The cells were trypsinized and centrifuged, and the medium was removed. The cells were re-suspended in PBS, centrifuged and PBS was removed carefully without disturbing the cell pellet. RNA was extracted from the treated cells using RNeasy® Plus Mini Kit (Qiagen) following the manufacturer's instructions. In brief, buffer RLT Plus (lysis buffer) was added to the cell pellet and homogenized. The homogenized lysate was transferred to a gDNA Eliminator spin column, centrifuged and the flow-through was saved. One volume of 70\% ethanol was added to the flowthrough, mixed and transferred to an RNeasy Mini spin column, centrifuged and the flow-through was discarded. Buffer RW1 (wash buffer containing guanidine salt, removing carbohydrates, proteins and fatty acids) was added to the column, centrifuged and the flow-through was discarded. Buffer RPE (wash buffer, removing traces of salts) was added to the column, centrifuged and the flow-through was discarded. This last step was repeated and the column was centrifuged again to further dry the membrane. RNase-free water was added to the column and centrifuged to elute the RNA. The quantity and purity of each RNA sample was assessed by measuring the absorbance at $260 \mathrm{~nm}$ using a Nanodrop spectrophotometer as well as using an Agilent® 2100 Bioanalyzer®. The samples were stored at $-80{ }^{\circ} \mathrm{C}$ and handed to the Functional Genomics Center Zurich (FGCZ), where library generation, sequencing and preliminary bioinformatic analyses were carried out.

mRNA library preparation for sequencing. The quality of the isolated RNA was determined with a Qubitß (1.0) Fluorometer (Life Technologies, California, USA) and a Bioanalyzer 2100 (Agilent, Waldbronn, Germany). Only those samples with a $260 / 280 \mathrm{~nm}$ ratio between $1.8-2.1$ and a 28S/18S ratio within $1.5-$ 2 were further processed. The Truseq RNA Sample Prep Kit v2 (Illumina, Inc, California, USA) was used in the succeeding steps. Briefly, total RNA samples (100-1000 ng) were poly A-enriched and then reverse-transcribed into double-stranded cDNA. The cDNA samples were fragmented, end-repaired and 
polyadenylated before ligation of TruSeq adapters containing the index for multiplexing. Fragments containing Truseq adapters on both ends were selectively enriched by PCR. The quality and quantity of the enriched libraries were validated using Qubit® (1.0) Fluorometer and the Caliper GX LabChip® GX (Caliper Life Sciences, Inc., USA). The product is a smear with an average fragment size of approximately $260 \mathrm{bp}$. The libraries were normalized to 10 $\mathrm{nM}$ in Tris-Cl $10 \mathrm{mM}, \mathrm{pH} 8.5$ with $0.1 \%$ Tween® 20.

Cluster generation and sequencing. The TruSeq PE Cluster Kit HS4000 or TruSeq SR Cluster Kit HS4000 (Illumina, Inc, California, USA) was used for cluster generation using $10 \mathrm{pM}$ of pooled normalized libraries on the CBOT. Sequencing was performed on the Illumina HiSeq 2000 paired end at 2101 bp or singlex101 bp or single end 100 bp using the TruSeq SBS Kit HS4000 (Illumina, Inc, California, USA).

Bioinformatic analysis. Reads were quality-checked with FasteC. Sequencing adapters were removed with Trimmomatic ${ }^{35}$ and reads were hard-trimmed by 5 bases at the $3^{\prime}$-end. Successively, reads at least 20 bases long, and with an overall average Phred quality score greater than 10 were aligned to the reference genome and transcriptome of Homo Sapiens (FASTA and GTF files, respectively, downloaded from GRCh38) with STAR v2.5.136 with default settings for single end reads. Distribution of the reads across genomic isoform expression was quantified using the $\mathrm{R}$ package GenomicRanges ${ }^{37}$ from Bioconductor Version 3.0. Differentially expressed genes were identified using the $R$ package edgeR ${ }^{38}$ from Bioconductor Version 3.0. A gene is marked as differentially expressed (DE) if it possesses the following characteristics: at least 10 counts in at least half of the samples in one group, $\mathrm{P} \leq 0.001$ and fold change $\geq 1.41$.

\section{Funding Sources}

This work was supported by ETH Zurich and the Swiss National Science Foundation (SNSF grant 200021_165551).

\section{Notes}

The authors declare no competing interests.

\section{ACKNOWLEDGMENTS}

We thank Mr. Elias Halabi (ETH Zurich) for providing the pLAMP1-mTurquoise2 plasmid, Mr. Zacharias Thiel (ETH Zurich) for providing the superoxide sensor probe HKSOX-1 and Dr. Bertran Rubi (ETH Zurich) for assistance with the 
LC-MS SIM method. We thank the Scientific Center for Optical and Electron Microscopy (ScopeM) at ETH Zurich for access to confocal microscopes. mRNA library generation, sequencing, and preliminary bioinformatics analyses were carried out at the Functional Genomics Center Zurich.

\section{REFERENCES}

(1) Jones, D. P. Redox Potential of GSH/GSSG Couple: Assay and Biological Significance. Methods Enzym. 2002, 348, 93-112. https://doi.org/10.1016/s0076-6879(02)48630-2.

(2) Jorgenson, T. C.; Zhong, W.; Oberley, T. D. Redox Imbalance and Biochemical Changes in Cancer. Cancer Res. 2013, 73, 6118-6123. https://doi.org/10.1158/0008-5472.CAN-13-1117.

(3) Hayden, M. R.; Sowers, J. R. Redox Imbalance in Diabetes. Antioxid. Redox Signal. 2007, 9, 865-867. https://doi.org/10.1089/ars.2007.1640.

(4) Smith, M. A.; Rottkampa, C. A.; Nunomura, A.; Raina, A. K.; Perry, G. Oxidative Stress in Alzheimer's Disease. Biochim. Biophys. Acta Mol. Basis Dis. 2000, 1502, 139-144. https://doi.org/10.1016/S0925-4439(00)00040-5.

(5) Jones, D. P.; Go, Y.-M. Redox Compartmentalization and Cellular Stress. Diabetes, Obes. Metab. 2010, 12, 116-125. https://doi.org/10.1111/j.1463-1326.2010.01266.x.

(6) Szendroedi, J.; Phielix, E.; Roden, M. The Role of Mitochondria in Insulin Resistance and Type 2 Diabetes Mellitus. Nat. Rev. Endocrinol. 2012, 8, 92-103. https://doi.org/10.1038/nrendo.2011.138.

(7) Lin, M. T.; Beal, M. F. F. Mitochondrial Dysfunction and Oxidative Stress in Neurodegenerative Diseases. Nature 2006, 443, 787-795. https://doi.org/10.1038/nature05292.

(8) Titov, D. V.; Cracan, V.; Goodman, R. P.; Peng, J.; Grabarek, Z.; Mootha, V. K. Complementation of Mitochondrial Electron Transport Chain by Manipulation of the NAD+/NADH Ratio. 2016, 352, 231-236. https://doi.org/10.1126/science.aad4017.

(9) Cracan, V.; Titov, D. V.; Shen, H.; Grabarek, Z.; Mootha, V. K. A Genetically Encoded Tool for Manipulation of NADP+/NADPH in Living Cells. Nat. Chem. Biol. 2017, 13, 1088-1095. https://doi.org/10.1038/nchembio.2454.

(10) Burns, J. A.; Butler, J. C.; Moran, J.; Whitesides, G. M. Selective Reduction of Disulfides by Tris(2-Carboxyethyl)Phosphine. J. Org. Chem. 1991, 56, 2648-2650. https://doi.org/10.1021/jo00008a014. 
(11) Tirla, A.; Rivera-Fuentes, P. Development of a Photoactivatable Phosphine Probe for Induction of Intracellular Reductive Stress with SingleCell Precision. Angew. Chem. Int. Ed. 2016, 55, 14709-14712. https://doi.org/10.1002/anie.201608779.

(12) Chevalier, A.; Zhang, Y.; Khdour, O. M.; Kaye, J. B.; Hecht, S. M. Mitochondrial Nitroreductase Activity Enables Selective Imaging and Therapeutic Targeting. J. Am. Chem. Soc. 2016, 138, 12009-12012. https://doi.org/10.1021/jacs.6b06229.

(13) Thiel, Z.; Rivera-Fuentes, P. Single-Molecule Imaging of Active Mitochondrial Nitroreductases Using a Photo-Crosslinking Fluorescent Sensor. Angew. Chem. Int. Ed. 2019, 58, 11474-11478. https://doi.org/10.1002/anie.201904700.

(14) Kim, G. J.; Lee, K.; Kwon, H.; Kim, H. J. Ratiometric Fluorescence Imaging of Cellular Glutathione. Org. Lett. 2011, 13, 2799-2801. https://doi.org/10.1021/o1200967w.

(15) Weerapana, E.; Wang, C.; Simon, G. M.; Richter, F.; Khare, S.; Dillon, M. B. D.; Bachovchin, D. A.; Mowen, K.; Baker, D.; Cravatt, B. F. Quantitative Reactivity Profiling Predicts Functional Cysteines in Proteomes. Nature 2010, 468, 790-797. https://doi.org/10.1038/nature09472.

(16) Zhang, H.; Limphong, P.; Pieper, J.; Liu, Q.; Rodesch, C. K.; Christians, E.; Benjamin, I. J. Glutathione-Dependent Reductive Stress Triggers Mitochondrial Oxidation and Cytotoxicity. FASEB J. 2012, 26, $1442-$ 1451. https://doi.org/10.1096/fj.11-199869.

(17) Hu, J. J.; Wong, N.-K.; Ye, S.; Chen, X.; Lu, M.-Y.; Zhao, A. Q.; Guo, Y.; Ma, A. C.-H.; Leung, A. Y.-H.; Shen, J.; et al. Fluorescent Probe HKSOX1 for Imaging and Detection of Endogenous Superoxide in Live Cells and in Vivo. J. Am. Chem. Soc. 2015, 137, 6837-6843. https://doi.org/10.1021/jacs.5b01881.

(18) Lange, P. S.; Chavez, J. C.; Pinto, J. T.; Coppola, G.; Sun, C.-W.; Townes, T. M.; Geschwind, D. H.; Ratan, R. R. ATF4 Is an Oxidative StressInducible, Prodeath Transcription Factor in Neurons in Vitro and in Vivo. J. Exp. Med. 2008, 205, 1227-1242. https://doi.org/10.1084/jem.20071460.

(19) Liberman, E. A.; Topaly, V. P.; Tsofina, L. M.; Jasaitis, A. A.; Skulachev, V. P. Mechanism of Coupling of Oxidative Phosphorylation and the Membrane Potential of Mitochondria. Nature 1969, 222, 1076-1078. https://doi.org/10.1038/2221076a0. 
(20) Riley, J. S.; Tait, S. W. G. Mechanisms of Mitophagy: Putting the Powerhouse into the Doghouse. Biol. Chem. 2016, 397, 617-635. https://doi.org/10.1515/hsz-2016-0137.

(21) Narendra, D.; Tanaka, A.; Suen, D.-F.; Youle, R. J. Parkin Is Recruited Selectively to Impaired Mitochondria and Promotes Their Autophagy. J. Cell Biol. 2008, 183, 795-803. https://doi.org/10.1083/jcb.200809125.

(22) Lemasters, J. J. Variants of Mitochondrial Autophagy: Types 1 and 2 Mitophagy and Micromitophagy (Type 3). Redox Biol. 2014, 2, 749-754. https://doi.org/10.1016/j.redox.2014.06.004.

(23) Halabi, E. A.; Pinotsi, D.; Rivera-Fuentes, P. Photoregulated Fluxional Fluorophores for Live-Cell Super-Resolution Microscopy with No Apparent Photobleaching. Nat. Commun. 2019, 10, 1232. https://doi.org/10.1038/s41467-019-09217-7.

(24) Insil, K.; Rodriguez-Enriquez, S.; Lemasters, J. J. Selective Degradation of Mitochondria by Mitophagy. Arch. Biochem. Biophys. 2007, 462, 245-253. https://doi.org/10.1016/j.abb.2007.03.034.

(25) Crawford, R. R.; Prescott, E. T.; Sylvester, C. F.; Higdon, A. N.; Shan, J.; Kilberg, M. S.; Mungrue, I. N. Human CHAC1 Protein Degrades Glutathione, and MRNA Induction Is Regulated by the Transcription Factors ATF4 and ATF3 and a Bipartite ATF/CRE Regulatory Element. J. Biol. Chem. 2015, 290, 15878-15891. https://doi.org/10.1074/jbc.M114.635144.

(26) Schröder, M.; Kaufman, R. J. The Mammalian Unfolded Protein Response. $\begin{array}{lllll}\text { Annu. Rev. Biochem. 2005, 739-789. } & \text { 74, }\end{array}$ https://doi.org/10.1146/annurev.biochem.73.011303.074134.

(27) Münch, C.; Harper, J. W. Mitochondrial Unfolded Protein Response Controls Matrix Pre-RNA Processing and Translation. Nature 2016, 534, $710-$ 713. https://doi.org/10.1038/nature18302.

(28) Fiesel, F. C.; James, E. D.; Hudec, R.; Springer, W.; Fiesel, F. C.; James, E. D.; Hudec, R.; Springer, W.; Fiesel, F. C.; James, E. D.; et al. Mitochondrial Targeted HSP90 Inhibitor Gamitrinib-TPP (G-TPP) Induces PINK1/Parkin-Dependent Mitophagy. Oncotarget 2017, 8, 106233-106248. https://doi.org/10.18632/oncotarget.22287.

(29) Quirós, P. M.; Prado, M. A.; Zamboni, N.; D’Amico, D.; Williams, R. W.; Finley, D.; Gygi, S. P.; Auwerx, J. Multi-Omics Analysis Identifies ATF4 as a Key Regulator of the Mitochondrial Stress Response in Mammals. J. Cell Biol. 2017, 216, 2027-2045. https://doi.org/10.1083/jcb.201702058. 
(30) Redler, R. L.; Wilcox, K. C.; Proctor, E. A.; Fee, L.; Caplow, M.; Dokholyan, N. V. Glutathionylation at Cys-111 Induces Dissociation of Wild Type and FALS Mutant SOD1 Dimers. Biochemistry 2011, 50, 7057-7066. https://doi.org/10.1021/bi200614y.

(31) Chen, M.; Wang, S.; Hsu, C.; Yin, P.; Yeh, T.-S.; Lee, H.; Tseng, L. CHAC1 Degradation of Glutathione Enhances Cystine-Starvation-Induced Necroptosis and Ferroptosis in Human Triple Negative Breast Cancer Cells via the GCN2-EIF2 $\alpha$-ATF4 Pathway. Oncotarget 2017, 8, 114588-114602. https://doi.org/10.18632/oncotarget.23055.

(32) Gibson, D. G.; Young, L.; Chuang, R.-Y.; Venter, J. C.; Hutchison, C. A.; Smith, H. O. Enzymatic Assembly of DNA Molecules up to Several Hundred Kilobases. Nat. Methods 2009, 6, 343-345. https://doi.org/10.1038/nmeth.1318.

(33) Goedhart, J.; von Stetten, D.; Noirclerc-Savoye, M.; Lelimousin, M.; Joosen, L.; Hink, M. A.; van Weeren, L.; Gadella Jr, T. W. J.; Royant, A. Structure-Guided Evolution of Cyan Fluorescent Proteins towards a Quantum Yield of 93\%. Nat. Commun. 2012, 3, 751. https://doi.org/10.1038/ncomms1738. (34) Zhang, M. S.; Brunner, S. F.; Huguenin-Dezot, N.; Liang, A. D.; Schmied, W. H.; Rogerson, D. T.; Chin, J. W. Biosynthesis and Genetic Encoding of Phosphothreonine through Parallel Selection and Deep Sequencing. Nat. Methods 2017, 14, 729-736. https://doi.org/10.1038/nmeth.4302.

(35) Bolger, A. M.; Lohse, M.; Usadel, B. Trimmomatic: A Flexible Trimmer for Illumina Sequence Data. Bioinformatics 2014, 30, 2114-2120. https://doi.org/10.1093/bioinformatics/btu170.

(36) Dobin, A.; Davis, C. A.; Schlesinger, F.; Drenkow, J.; Zaleski, C.; Jha, S.; Batut, P.; Chaisson, M.; Gingeras, T. R. STAR: Ultrafast Universal RNA-Seq Aligner. Bioinformatics 2013, 29, $15-21$. https://doi.org/10.1093/bioinformatics/bts635.

(37) Lawrence, M.; Huber, W.; Hervé, P.; Aboyoun, P.; Carlson, M.; Gentleman, R.; Morgan, M. T.; Carey, V. J. Software for Computing and Annotating Genomic Ranges. PLOS Comput. Biol.S Comput. Biol. 2013, 9, e1003118. https://doi.org/10.1371/journal.pcbi.1003118.

(38) Robinson, M. D.; McCarthy, D. J.; Smyth, G. K. EdgeR: A Bioconductor Package for Differential Expression Analysis of Digital Gene Expression Data. Bioinformatics 2010, 26, 139-140. https://doi.org/10.1093/bioinformatics/btp616. 


\section{Disruption of Redox Homeostasis by Enzymatic Activation of a Trialkylphosphine Probe in Mitochondria}

Jade Nguyen ${ }^{\mathrm{a}, \mathrm{b}}$, Alina Tirla ${ }^{\mathrm{a}}$ and Pablo Rivera-Fuentes ${ }^{\mathrm{a}, \mathrm{b}^{*}}$

aLaboratory of Organic Chemistry, ETH Zurich, 8093, Zurich, Switzerland.

bInstitute of Chemical Sciences and Engineering, EPF Lausanne, 1015, Lausanne, Switzerland.

*pablo.riverafuentes@epfl.ch

\section{Contents}

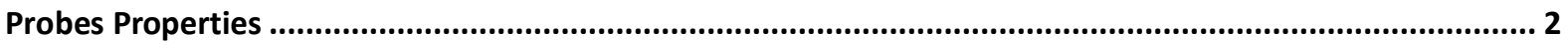

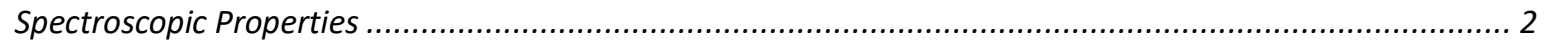

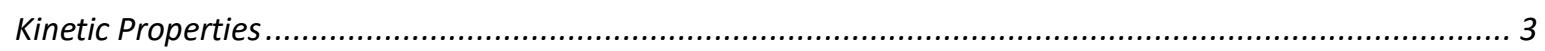

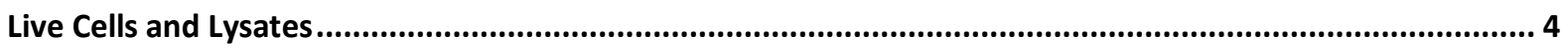

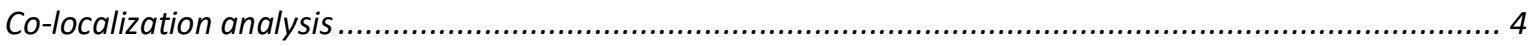

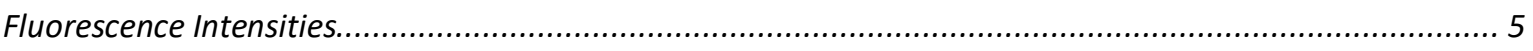

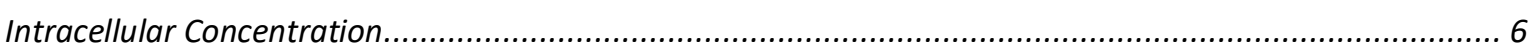

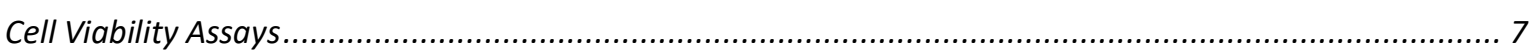

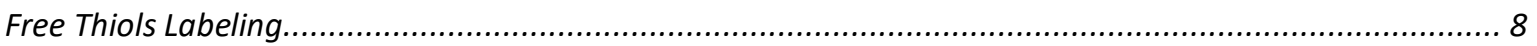

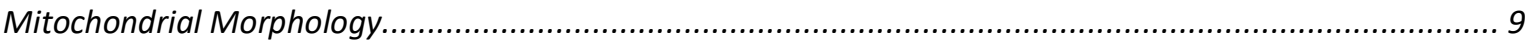

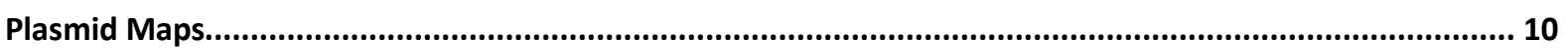

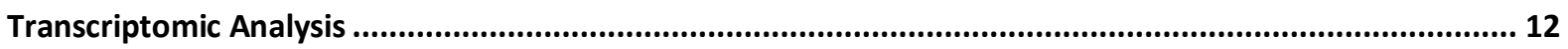

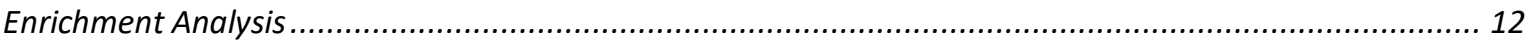

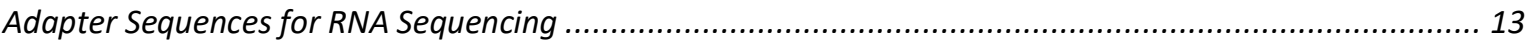

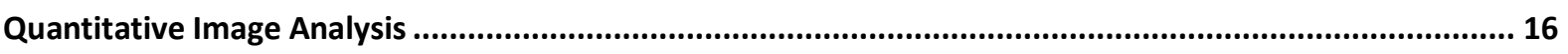

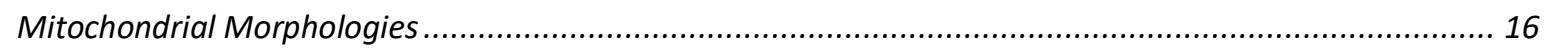

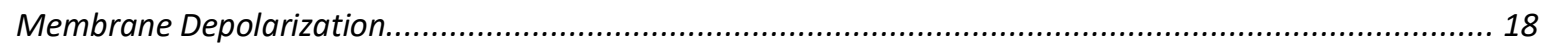

Co-localization of LC3 punctae and Mitochondria ..................................................................... 19

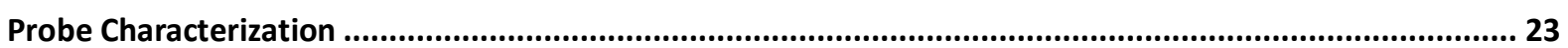

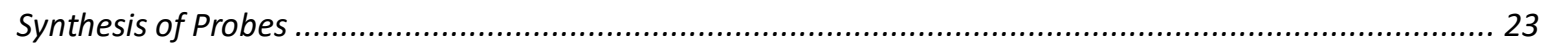

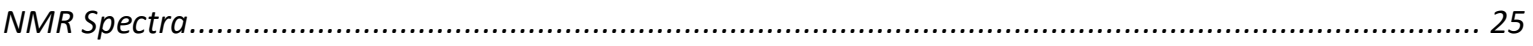




\section{Probes Properties}

Spectroscopic Properties

\begin{tabular}{|c|c|c|c|c|c|}
\hline & $\begin{array}{c}\varepsilon \\
\left(\mathrm{M}^{-1} \mathrm{~cm}^{-1}\right)\end{array}$ & $\begin{array}{c}\lambda_{\text {max abs }} \\
(\mathrm{nm})\end{array}$ & $\begin{array}{c}\phi_{\mathrm{em}} \\
(\%)\end{array}$ & $\begin{array}{c}\lambda_{\text {max }, \mathrm{em}} \\
(\mathrm{nm})\end{array}$ & $\begin{array}{c}\varepsilon \times \phi_{\mathrm{em}} \\
\left(\mathrm{M}^{-1} \mathrm{~cm}^{-1}\right)\end{array}$ \\
\hline probe 1 & 27840 & 285 & 0 & - & - \\
\hline probe 3 & 19627 & 378 & 0 & - & - \\
\hline probe 2 & 38416 & 481 & 0.18 & 555 & 6915 \\
\hline
\end{tabular}

Table S1. Photophysical properties of probes measured in PBS $(5 \mu \mathrm{M})$.
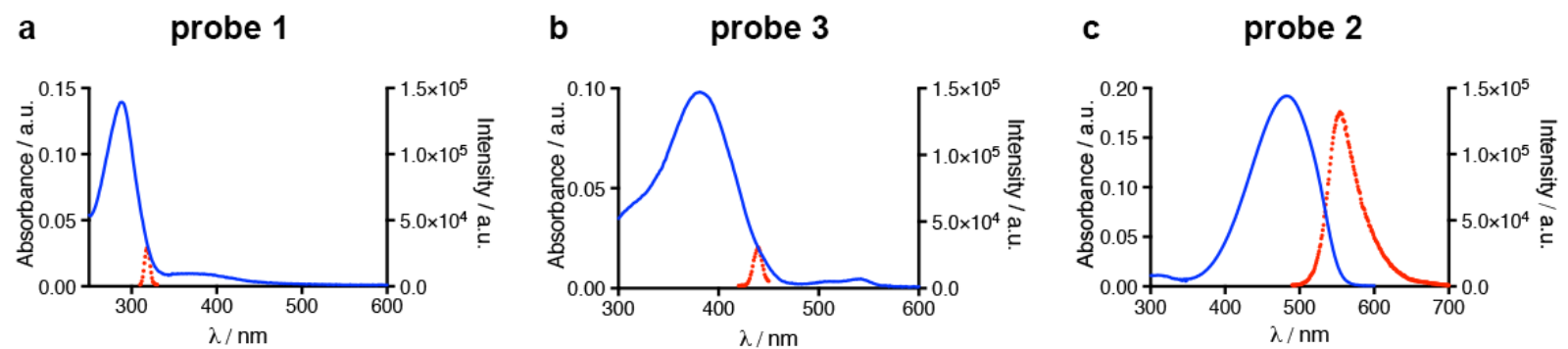

Figure S1. Absorption and fluorescence spectra of the probes 1, 2 and 3. Absorption (blue) and fluorescence (red) spectra of the probes $(5 \mu \mathrm{M})$ in phosphate buffered saline (PBS). a, Spectra of probe 1. b, Spectra of probe 3. c, Spectra of probe 2. 


\section{Kinetic Properties}

a

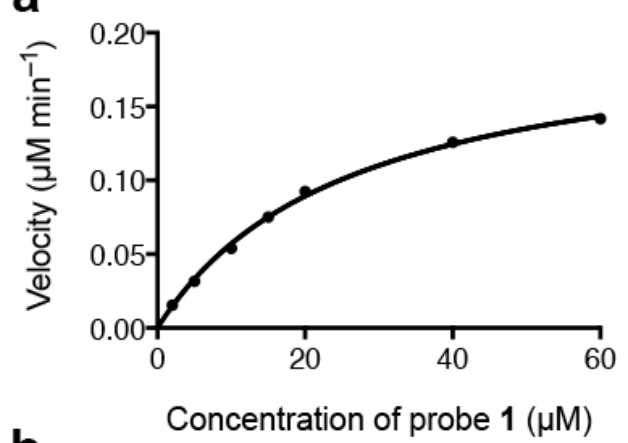

$R^{2}=0.998$

$V_{\text {max }}=0.206 \mu \mathrm{M} \mathrm{min}^{-1}$

$\mathrm{K}_{\mathrm{m}}=26.09 \mu \mathrm{M}$

$k_{\text {cat }}=2.47 \mathrm{~min}^{-1}$

$k_{\text {cat }} / \mathrm{K}_{\mathrm{m}}=0.095 \mu \mathrm{M}^{-1} \mathrm{~min}^{-1}$

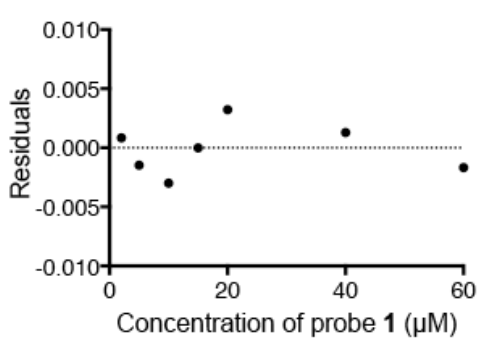

b

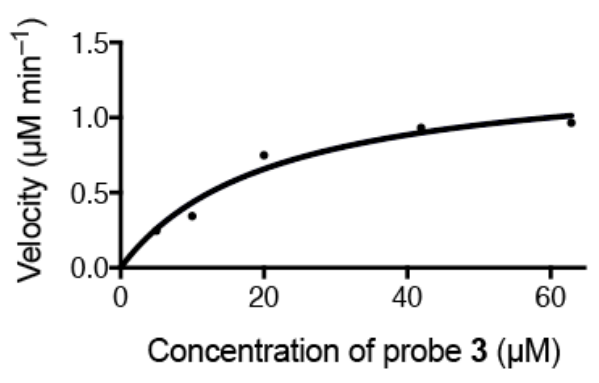

$\mathrm{R}^{2}=0.96$

$\mathrm{V}_{\text {max }}=1.35 \mu \mathrm{M} \mathrm{min}^{-1}$

$\mathrm{K}_{\mathrm{m}}=21.2 \mu \mathrm{M}$

$k_{\text {cat }}=326 \mathrm{~min}^{-1}$

$k_{\text {cat }} / \mathrm{K}_{\mathrm{m}}=15.4 \mu \mathrm{M}^{-1} \mathrm{~min}^{-1}$

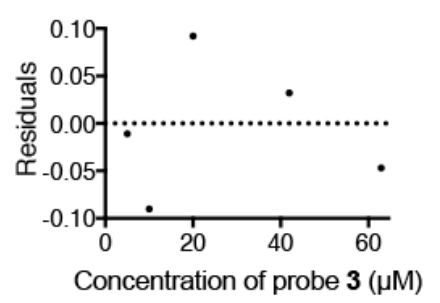

Figure S2. Michaelis-Menten kinetics of activation of probes 1 and 3 by bacterial nitroreductase (E. coli $\mathrm{B}$ nitroreductase). a, Activation of probe 1. b, Activation of probe 3. In both cases, the absorbance of reported dye 2 was followed, giving the overall kinetics of reduction of the nitro group to amine, and release of phosphine (extremely fast step) for compound 1 . 


\section{Live Cells and Lysates}

\section{Co-localization analysis}

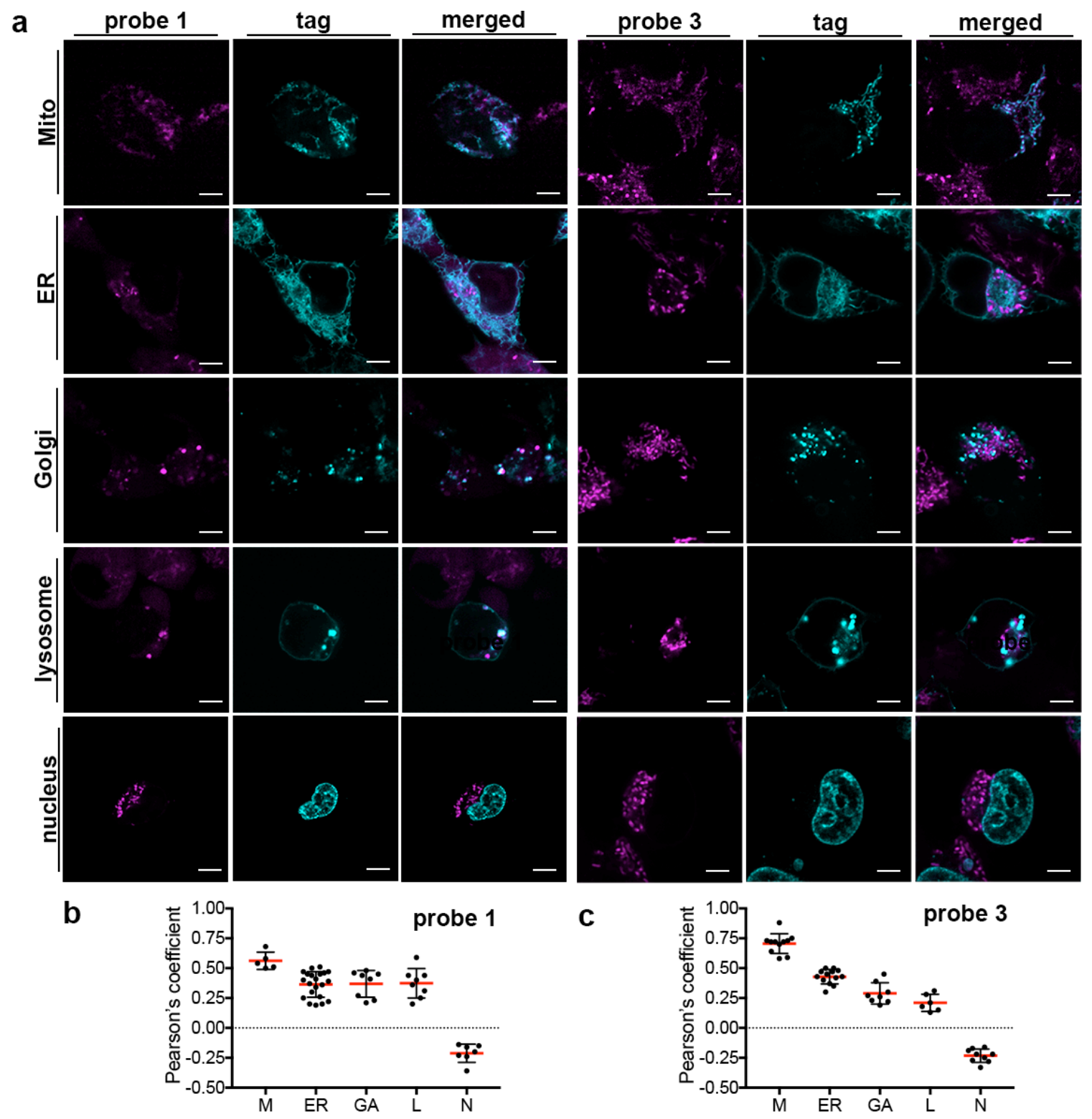

Figure S3. Co-localization of reporter dye 2 and organelle-targeted

mTurquoise2. a, HEK293 cells were incubated with Hoechst dye $(5 \mu \mathrm{M})$, transfected with plasmids pmT-ER, pmT-Golgi or pmT-Lyso. The cells were incubated with probe 1 (left) and 3 (right). Scale bar $=10 \mu \mathrm{m}$. b, Pearson's correlation coefficients of probe $1, n>5$ cells from three biological replicates. c, Pearson's correlation coefficients of probe $3, n>6$ cells from three biological replicates. For both probes, reporter dye $\mathbf{2}$ is generated in mitochondria. 
Fluorescence Intensities

a

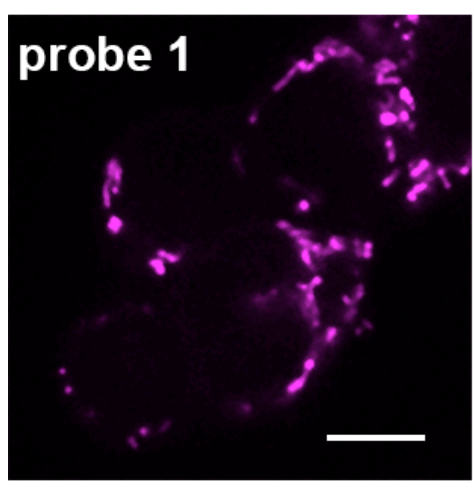

b

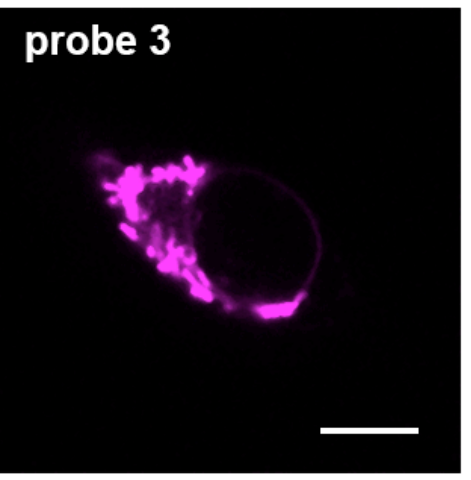

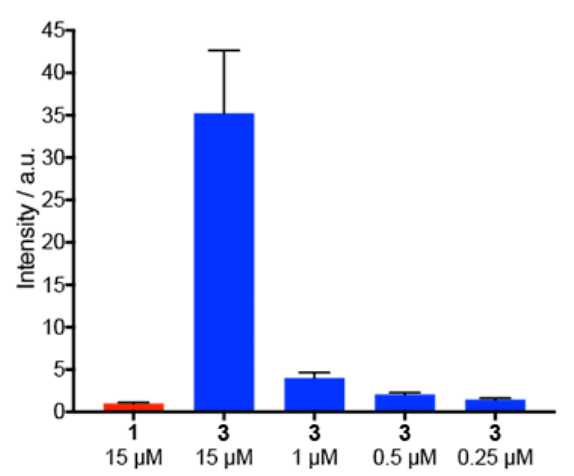

Figure S4. Relative fluorescent intensities of intracellular activation of probes

1 and 3. a, Intracellular fluorescence upon enzymatic activation of compounds 1 $(15 \mu \mathrm{M})$ or $3(0.5 \mu \mathrm{M})$ in live HEK293 cells. Fluorescence intensity was measured in the red channel (excitation: $561 \mathrm{~nm}$, identical settings for both compounds). Scale bar $=10 \mu \mathrm{m} . \mathbf{b}$, Relative fluorescence intensities of probe $\mathbf{3}$ at concentrations ranging from $15 \mu \mathrm{M}$ to $0.25 \mu \mathrm{M}$ (blue) compared to probe 1 at $15 \mu \mathrm{M}$ (red) after $1 \mathrm{~h}$ incubation. Bars indicate average of relative intensity of cells when treated with $\mathbf{3}$, relative to 1 at $15 \mu \mathrm{M}$ (intensity $=1$ ). Measurements of $n>10$ cells from biological replicates. Error bars represent 95\% confidence ranges. 
Intracellular Concentration

a

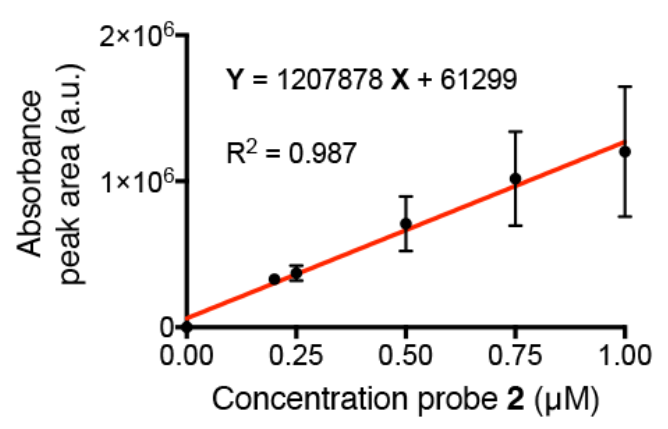

b

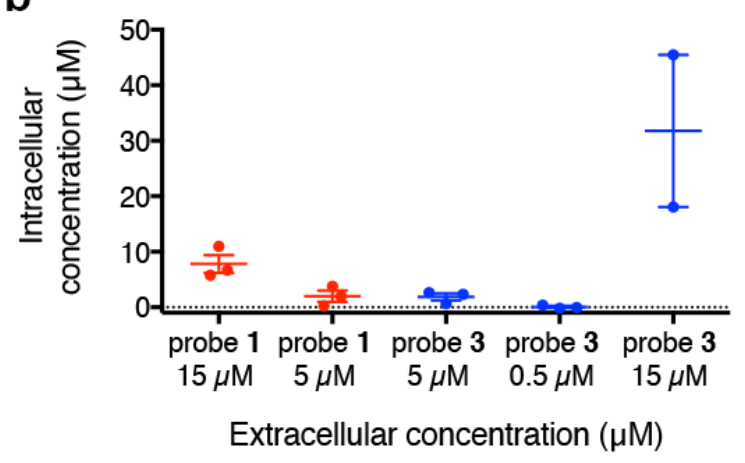

Figure S5. Intracellular concentrations of reporter dye 2 upon treatment of cells with probes 1 or 3, measured by LC-MS SIM. a, Calibration curve. Control lysates spiked with probe $\mathbf{2}$ at the indicated concentrations. Absorbance peak areas were used to determine concentrations. b, Intracellular concentrations of probes 1 and $\mathbf{5}$ after incubation at the indicated extracelullar concentrations for $3 \mathrm{~h}$. Lysates and calibration curves are from three biological replicates $(n=3)$, error bars are standard deviation of the mean. This experiment reveals that at extracellular concentrations of $15 \mu \mathrm{M}$, the intracellular production of 2 from 3 is much greater than from probe 1, in agreement with the more intense fluorescence observed in the microscopy experiment (Figure S4). Aiming at finding a compromise between the fluorescence and LC-MS experiments, we decided that an extracellular concentration of $15 \mu \mathrm{M}$ of 1 and $5 \mu \mathrm{M}$ of 3 gives roughly equivalent intracellular concentrations of 2. 


\section{Cell Viability Assays}
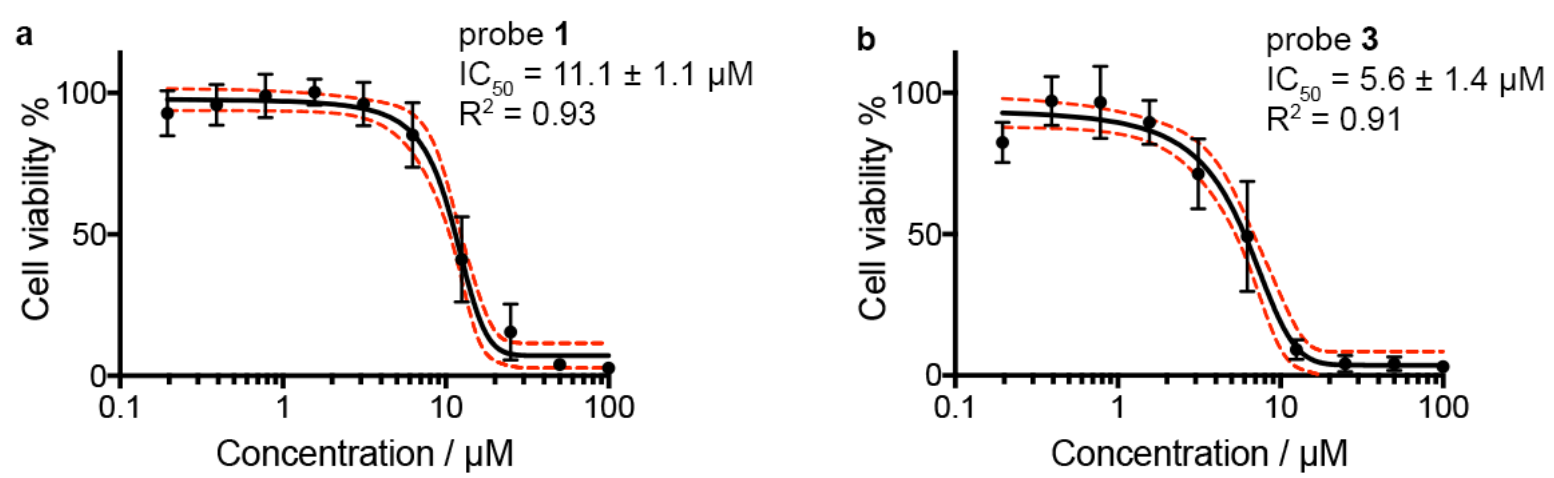

Figure S6. Cell viability assays. HEK293 cells were incubated with a, probe $\mathbf{1}$ or $\mathbf{b}$, probe 3 at concentrations ranging from $100 \mathrm{nM}$ to $100 \mu \mathrm{M}$ for $48 \mathrm{~h}$. The cells were then incubated with MTT solution and the absorbance of formazan was measured at $550 \mathrm{~nm}$. DMSO control $(0.5 \%)$ sets the $100 \%$ viability. Black points are averages of nine measurements (triplicates of triplicates). Black lines are four parameters logistic regression (4PL) fit. Error bars represent 95\% confidence ranges. Red dashed lines are $95 \%$ confidence interval bands. 


\section{Free Thiols Labeling}

a
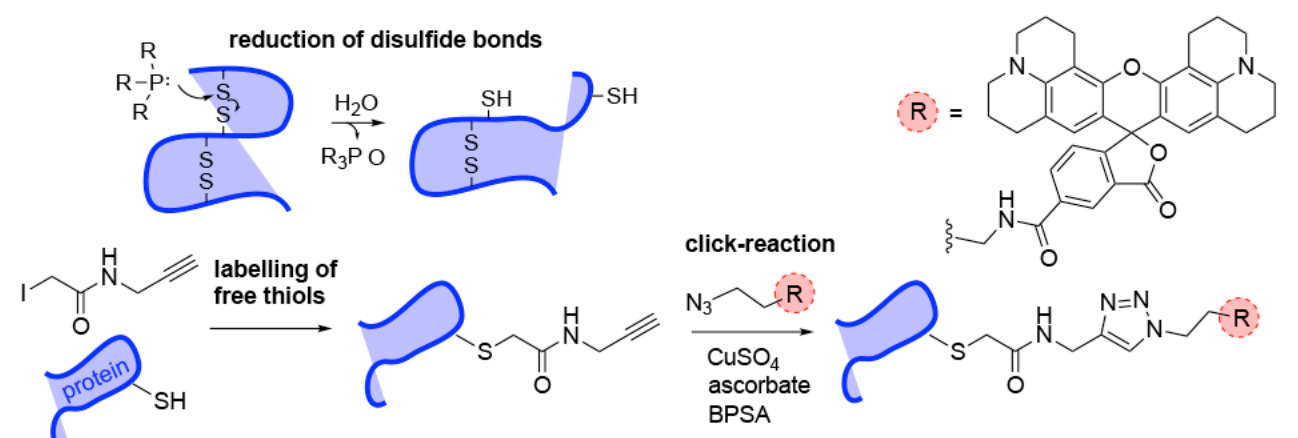

b
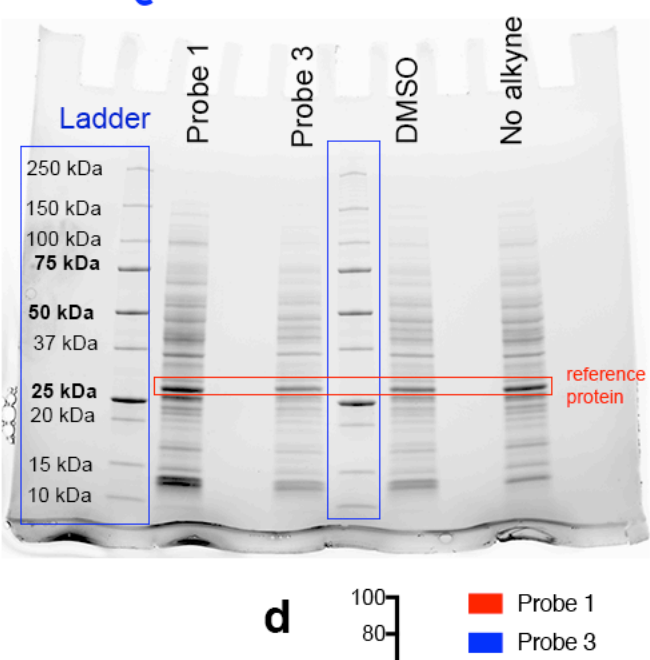

C

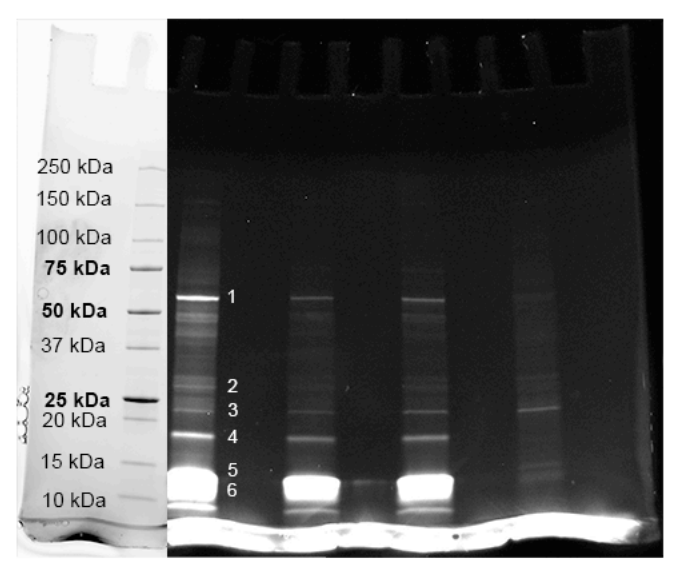

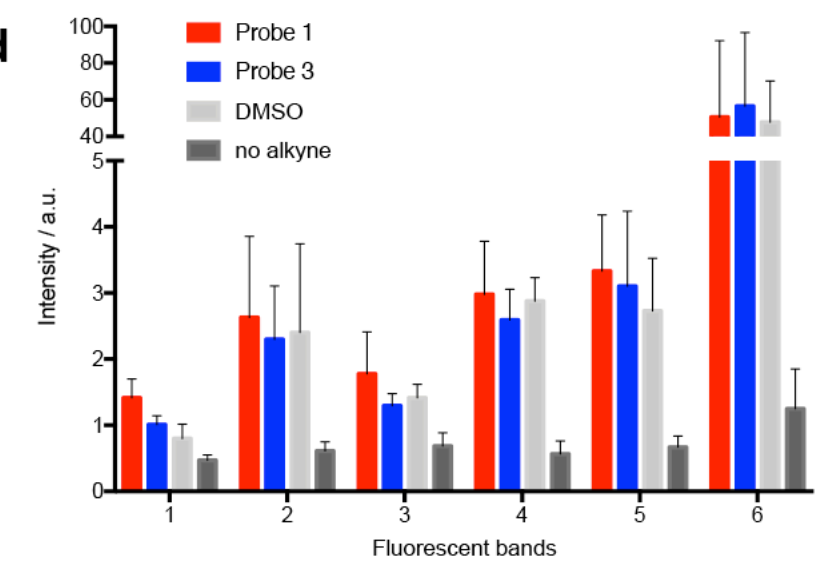

Figure S7. Tributylphosphine does not reduce disulfide bonds in proteins. a, Mechanism of action of proteins disulfide bonds reduction by trialkylphosphines and experimental procedure of labelling of free thiols by iodoacetamide alkyne (IAA) in cell lysates followed by click-reaction with a fluorescent reporter. $\mathbf{b}$, image of stainfree gel and c, fluorescent image (same gel), of HEK293 whole cell lysates treated with probe $1(15 \mu \mathrm{M})$, probe $3(5 \mu \mathrm{M})$ and DMSO $(0.5 \%)$ for $3 \mathrm{~h}$, lysed and immediately labelled with IAA, followed by click-reaction with fluorescent reporter. 
The protein ladder (b, blue rectangle) is reported on the fluorescent image (c) for ease of observation. The reference protein (b, red rectangle) is used to normalize the band intensities in each sample based on the stain-free gel image. The bands quantified are numbered (c, white numbers). d, quantification of fluorescence intensity. Measurements were carried out in biological triplicates. Bars represent means and error bars represent $95 \%$ confidence intervals. Statistical significance was assessed by unpaired, two-tailed, Mann-Whitney test. None of the comparisons displayed statistical significance, $P$-values $>0.05$.

\section{Mitochondrial Morphology}
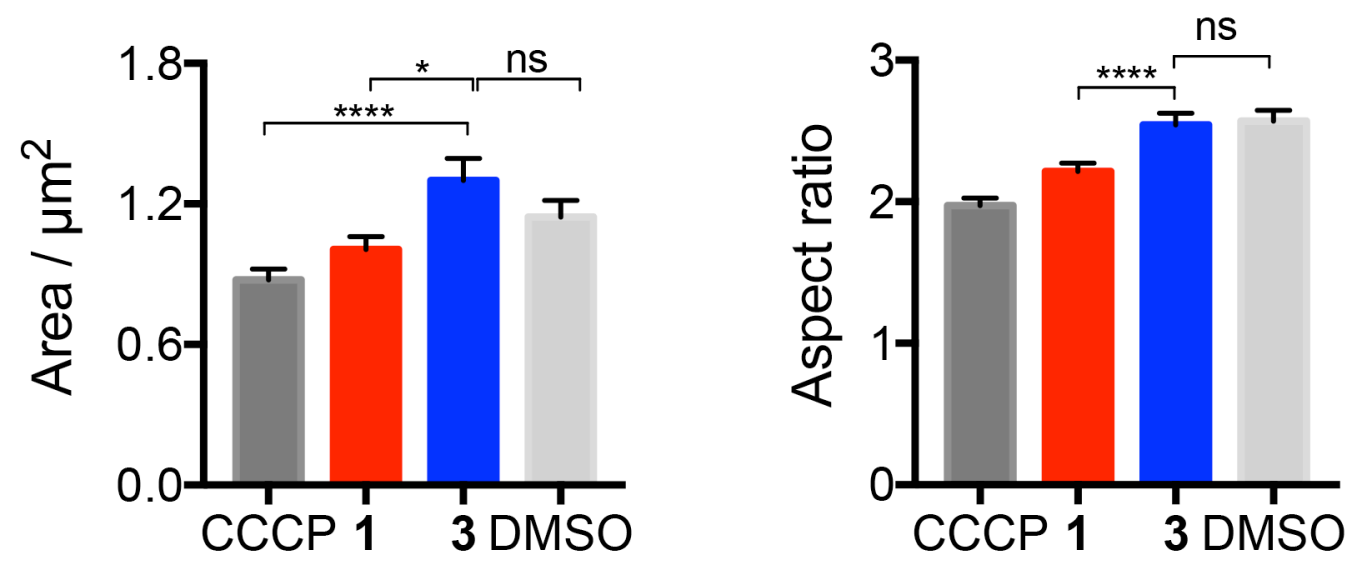

Figure S8. Analysis of mitochondrial shapes after treatment with CCCP $(20 \mu \mathrm{M}$, $2 \mathrm{~h})$, probe $1(15 \mu \mathrm{M}, 3-4 \mathrm{~h})$, probe $3(5 \mu \mathrm{M}, 3-4 \mathrm{~h})$, or DMSO $(1 \%, 3-4 \mathrm{~h})$. Measurements were carried out in biological triplicates and morphological data from $n=1000$ mitochondria were employed for each condition. Means are plotted and errors bars represent $95 \%$ confidence ranges. Statistical significance was assessed by unpaired, two-tailed, Mann-Whitney test. $P$ values: ${ }^{* \star * *}<0.0001,{ }^{*}<0.05$, ns $=$ not significant $>0.05$. 


\section{Plasmid Maps}

Plasmid maps of assembled pmTurquoise2-Parkin and pmTurquoise2-LC3 are shown in Figure S9 and primers used in Table S2.
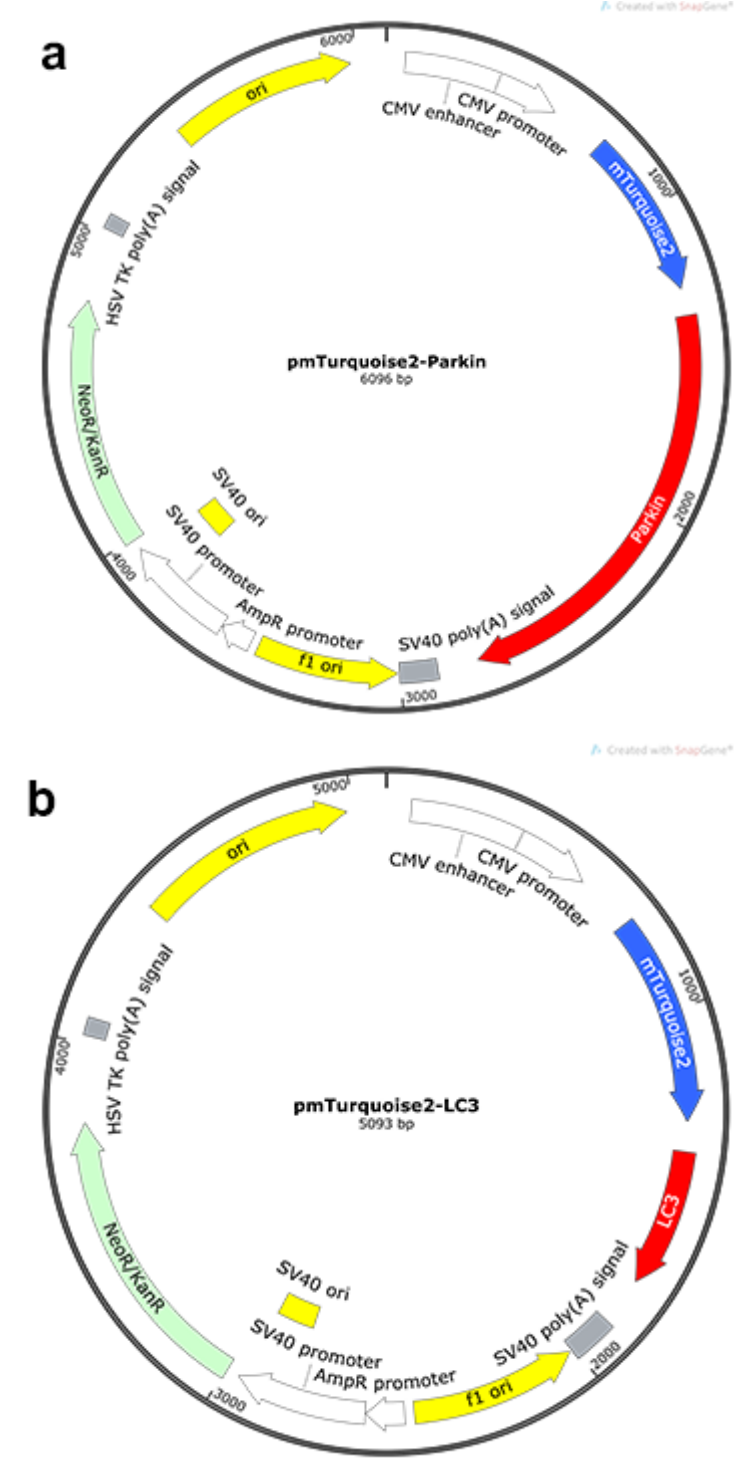

Figure S9. Plasmid maps of Gibson assembly constructs. pmTurquoise2 fluorescent tags are depicted in blue and the proteins of interest in red. General features, selective markers and promoters are also depicted on the maps. a, pmTurquoise2Parkin: mammalian expression of Parkin fused to mTurquoise2. $\mathbf{b}$, pmTurquoise2LC3: mammalian expression of LC3 fused to mTurquoise2. Maps were generated using SnapGene ${ }^{\circledR}$ 4.1.9. 
Table S2. PCR primers used for the Gibson assemblies of pmTurquoise2-LC3 and pmTurquoise2-Parkin.

\begin{tabular}{|c|c|c|c|}
\hline & primer name & direction & primer sequence: $5^{\prime}-$ to $-3^{\prime}$ \\
\hline \multirow{4}{*}{ 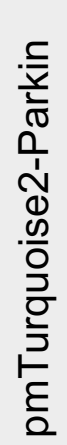 } & pmt-PARK-BB.for & forward & GCG CGA TCA CAT GGT CCT G \\
\hline & pmt-PARK-BB.rev & reverse & GCA GAT GAA CTT CAG GGT CA \\
\hline & PMT-park-INS.for & forward & CCC TGA AGT TCA TCT GCA CCA C \\
\hline & PMT-park-INS.rev & reverse & GGA CCA TGT GAT CGC TTC TC \\
\hline \multirow{4}{*}{ 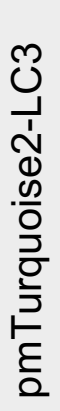 } & pmt-LC3-BB.for & forward & СCT GCT GGA GTT CGT GAC C \\
\hline & pmt-LC3-BB.rev & reverse & GGT CAG CTT GCC GTA GGT G \\
\hline & PMT-Ic3-INS.for & forward & CTA CGG CAA GCT GAC CCT GA \\
\hline & PMT-Ic3-INS.rev & reverse & CAG GAA CTC CAG CAG GAC CA \\
\hline
\end{tabular}




\section{Transcriptomic Analysis}

\section{Enrichment Analysis}
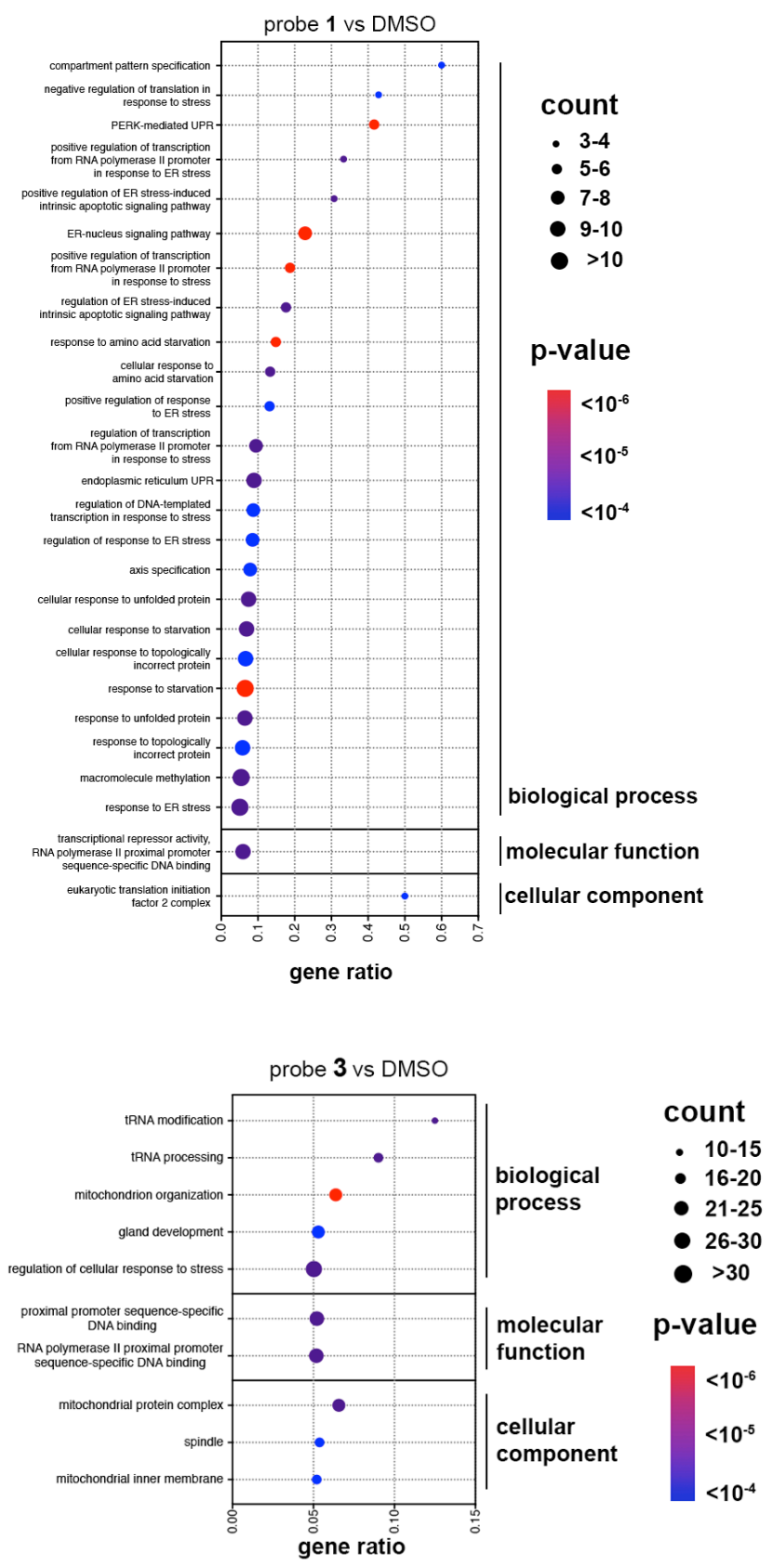

Figure S10. Enrichment analysis of significantly upregulated or downregulated genes in HEK293 cells using gene ontology (GO) database. a, Cells treated with probe $1(15 \mu \mathrm{M})$ compared to DMSO control. b, Cells treated with probe $3(5 \mu \mathrm{M})$ and compared to DMSO control. Size of circle represents the total number of genes associated with the function or cellular component. Gene ratio refers to the number 
of genes that are differentially regulated divided by the total genes known to participate in a specific function or cellular component.

\section{Adapter Sequences for RNA Sequencing}

Oligonucleotide sequences for TruSeq ${ }^{\mathrm{TM}}$ RNA and DNA Sample Prep Kits

TruSeq Universal Adapter

5'-AATGATACGGCGACCACCGAGATCTACACTCTTTCCCTACACGACGCTCTTC CGATCT

TruSeq $^{\mathrm{TM}}$ Adapters

TruSeq Adapter, Index 1

5'-GATCGGAAGAGCACACGTCTGAACTCCAGTCACATCACGATCTCGTATGCC GTCTTCTGCTTG

TruSeq Adapter, Index 2

5'-GATCGGAAGAGCACACGTCTGAACTCCAGTCACCGATGTATCTCGTATGCC GTCTTCTGCTTG

TruSeq Adapter, Index 3

5'-GATCGGAAGAGCACACGTCTGAACTCCAGTCACTTAGGCATCTCGTATGCC GTCTTCTGCTTG

TruSeq Adapter, Index 4

5'-GATCGGAAGAGCACACGTCTGAACTCCAGTCACTGACCAATCTCGTATGCC GTCTTCTGCTTG

TruSeq Adapter, Index 5

5'-GATCGGAAGAGCACACGTCTGAACTCCAGTCACACAGTGATCTCGTATGCC GTCTTCTGCTTG

TruSeq Adapter, Index 6

5'-GATCGGAAGAGCACACGTCTGAACTCCAGTCACGCCAATATCTCGTATGCC GTCTTCTGCTTG

TruSeq Adapter, Index 7

5'-GATCGGAAGAGCACACGTCTGAACTCCAGTCACCAGATCATCTCGTATGCC GTCTTCTGCTTG

TruSeq Adapter, Index 8

5'-GATCGGAAGAGCACACGTCTGAACTCCAGTCACACTTGAATCTCGTATGCCG TCTTCTGCTTG 
TruSeq Adapter, Index 9

5'-GATCGGAAGAGCACACGTCTGAACTCCAGTCACGATCAGATCTCGTATGCC GTCTTCTGCTTG

TruSeq Adapter, Index 10

5'-GATCGGAAGAGCACACGTCTGAACTCCAGTCACTAGCTTATCTCGTATGCCG TCTTCTGCTTG

TruSeq Adapter, Index 11

5'-GATCGGAAGAGCACACGTCTGAACTCCAGTCACGGCTACATCTCGTATGCC GTCTTCTGCTTG

TruSeq Adapter, Index 12

5'-GATCGGAAGAGCACACGTCTGAACTCCAGTCACCTTGTAATCTCGTATGCCG TCTTCTGCTTG

TruSeq Adapter, Index 13

5'-GATCGGAAGAGCACACGTCTGAACTCCAGTCACAGTCAACAATCTCGTATG CCGTCTTCTGCTTG

TruSeq Adapter, Index 14

5'-GATCGGAAGAGCACACGTCTGAACTCCAGTCACAGTTCCGTATCTCGTATG CCGTCTTCTGCTTG

TruSeq Adapter, Index 15

5'-GATCGGAAGAGCACACGTCTGAACTCCAGTCACATGTCAGAATCTCGTATG CCGTCTTCTGCTTG

TruSeq Adapter, Index 16

5'-GATCGGAAGAGCACACGTCTGAACTCCAGTCACCCGTCCCGATCTCGTATG CCGTCTTCTGCTTG

TruSeq Adapter, Index 184

5'-GATCGGAAGAGCACACGTCTGAACTCCAGTCACGTCCGCACATCTCGTATG CCGTCTTCTGCTTG

TruSeq Adapter, Index 19

5'-GATCGGAAGAGCACACGTCTGAACTCCAGTCACGTGAAACGATCTCGTATG CCGTCTTCTGCTTG

TruSeq Adapter, Index 20

5'-GATCGGAAGAGCACACGTCTGAACTCCAGTCACGTGGCCTTATCTCGTATG CCGTCTTCTGCTTG

TruSeq Adapter, Index 21 
5'-GATCGGAAGAGCACACGTCTGAACTCCAGTCACGTTTCGGAATCTCGTATG CCGTCTTCTGCTTG

TruSeq Adapter, Index 22

5'-GATCGGAAGAGCACACGTCTGAACTCCAGTCACCGTACGTAATCTCGTATG CCGTCTTCTGCTTG

TruSeq Adapter, Index 23

5'-GATCGGAAGAGCACACGTCTGAACTCCAGTCACGAGTGGATATCTCGTATG CCGTCTTCTGCTTG

TruSeq Adapter, Index 25

5'-GATCGGAAGAGCACACGTCTGAACTCCAGTCACACTGATATATCTCGTATGC CGTCTTCTGCTTG

TruSeq Adapter, Index 27

5'-GATCGGAAGAGCACACGTCTGAACTCCAGTCACATTCCTTTATCTCGTATGC CGTCTTCTGCTTG 


\section{Quantitative Image Analysis}

\section{Mitochondrial Morphologies}

Mitochondrial morphology of live HEK293 cells was assessed by quantification of the signals obtained from activation of either probe 1 and 3 . Cells were treated with 1 $(15 \mu \mathrm{M})$ or $3(5 \mu \mathrm{M})$ for 3-4 h. For the controls, CCCP $(20 \mu \mathrm{M}, 2 \mathrm{~h})$ or DMSO (3-4 h), mitochondria were stained with MitoTracker Deep Red (MDR). Good quality images, with strong signal but avoiding saturation, were collected in the red $\left(\lambda_{\mathrm{ex}}=561 \mathrm{~nm}\right.$; $\left.\lambda_{\mathrm{em}}=630 \pm 75 \mathrm{~nm}\right)$ and far-red $\left(\lambda_{\mathrm{ex}}=640 \mathrm{~nm} ; \lambda_{\mathrm{em}}=700 \pm 75 \mathrm{~nm}\right)$ imaging channels. The images were collected using a $100 \times$ objective (100× 1.49 CFI Apo TIRF), an EMCCD camera (Digital Orca Flash 4.0 V2, rolling shutter usage, $1024 \times 1024$ pixel (pixel size $13 \mu \mathrm{m}$ ) with no binning $(1 \times 1)$. Representative example of red images is displayed in Figure S11a. Binary images were generated by segmentation of red images. The following macro was applied in Fiji (ImageJ):

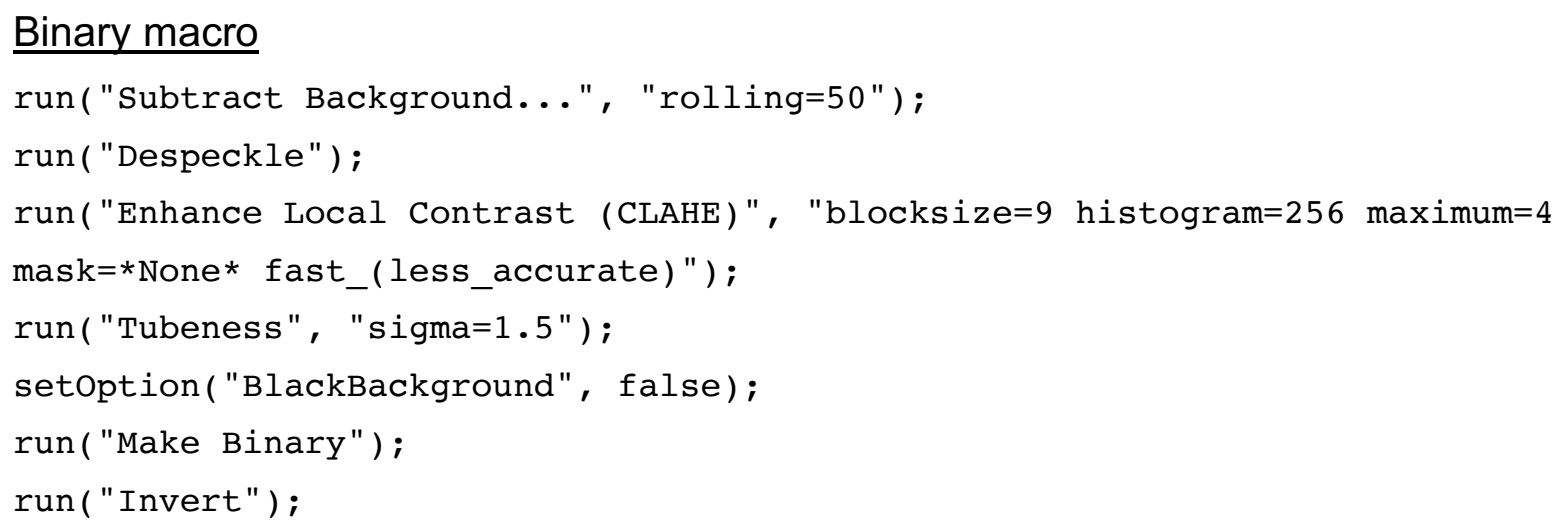

Application of this macro generated binary images of mitochondria. Representative examples are displayed in Figure S11b. The shapes of mitochondria were analyzed using the "analyze particle" plugin from Fiji software. The scale was set and a centered region of interest of $800 \times 800$ pixels was chosen to run the analysis, to avoid taking into account less intense signals on the edges of the frames. The following macro was applied in Fiji (ImageJ): 


\section{Scale macro}

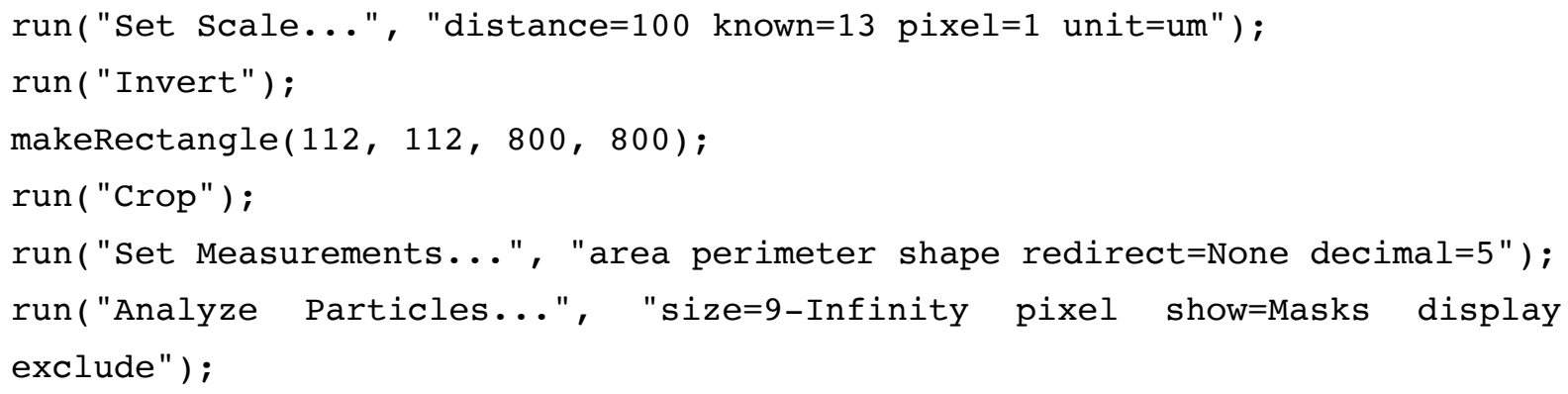

The circularity, area and aspect ratio values for each mitochondrion were obtained from these measurements. Representative examples of obtained binary images are displayed in Figure S11c. The lengths of mitochondria were calculated using the scaled binary images and skeletonize plugin from Fiji. The skeletonize plugin thins mitochondria to be 1 pixel wide. The "analyze particle" plugin was used to determine the length of mitochondria, as the area of skeletonized mitochondria represent the total length of mitochondria in pixel. The following macro was applied in Fiji (ImageJ):

\section{Skeletonize macro}

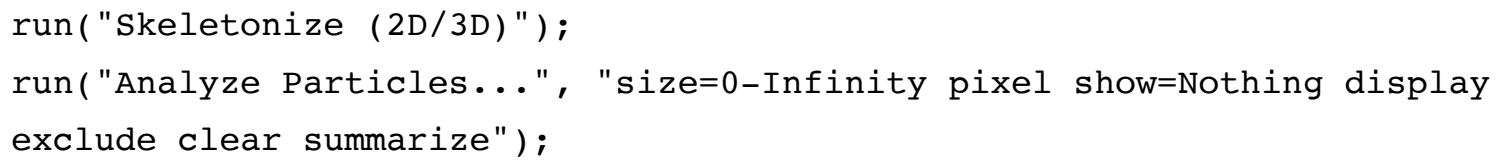

Representative example of skeletonized image is displayed in Figure S11d. The length values for each mitochondrion were obtained from these measurements.
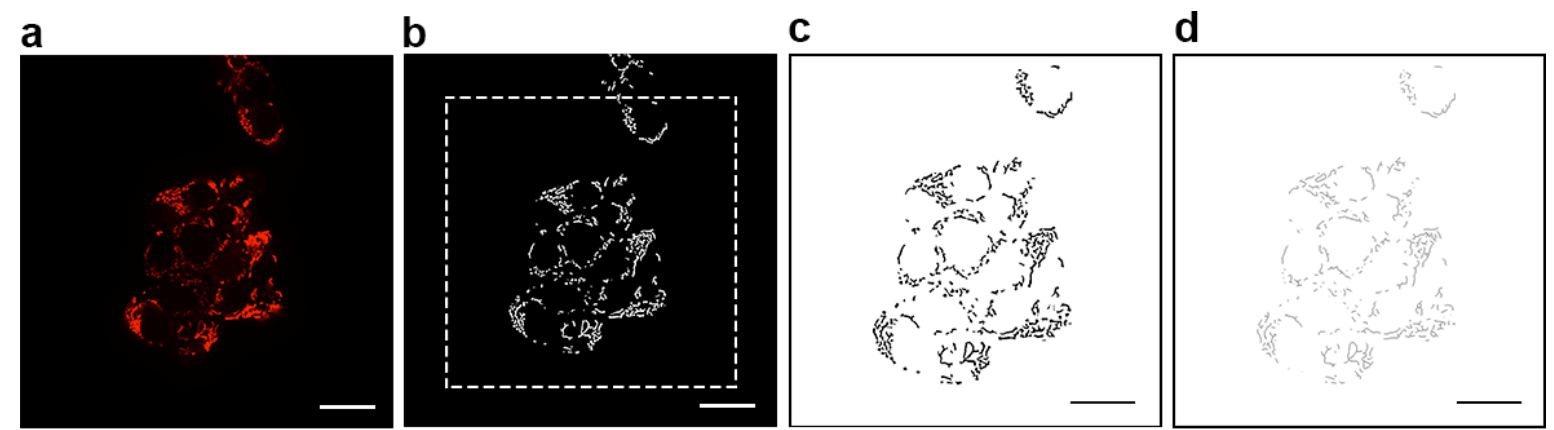

Figure S11. a, Example of red image of mitochondria. Scale bar $=20 \mu \mathrm{m}$. b, Segmented, binary image of mitochondria obtained employing "binary macro" on image a. c, Binary images were scaled and analyzed employing "scale macro" on image b. d, Binary image was skeletonized and analyzed employing "skeletonize macro" on image $c$. 


\section{Membrane Depolarization}

Membrane depolarization of live HEK293 cells was assessed by quantification and localization of the signals obtained from activation of either probe 1 and 5 as well as CCCP stained with probe 5. Cells were treated with $1(15 \mu \mathrm{M}), 5(1 \mu \mathrm{M})$ or CCCP $(20 \mu \mathrm{M})$ co-incubated with 5 for $2 \mathrm{~h}$. Good quality images, with strong signal but avoiding saturation, were collected in the red $\left(\lambda_{\mathrm{ex}}=561 \mathrm{~nm}\right)$ imaging channel. The images were collected using a $60 \times$ objective (Silicon UPlanSApo $60 \times$ NA = 1.3), 4 GaAsP PMTs (spectral detection option) and a transmission PMT $(800 \times 800$ pixel, pixel size $7.5 \mu \mathrm{m})$. Representative example of red images is displayed in Figure S10a. A cell was chosen as region of interest. A pixel intensity threshold was set to differentiate the mitochondrial (Figure S12b) from the cytoplasmic fraction (Figure S12c). In a similar manner, a pixel intensity threshold was set to determine signal from noise (Fig. S12d). The following macros were applied in Fiji (ImageJ) after which the measure command was run:

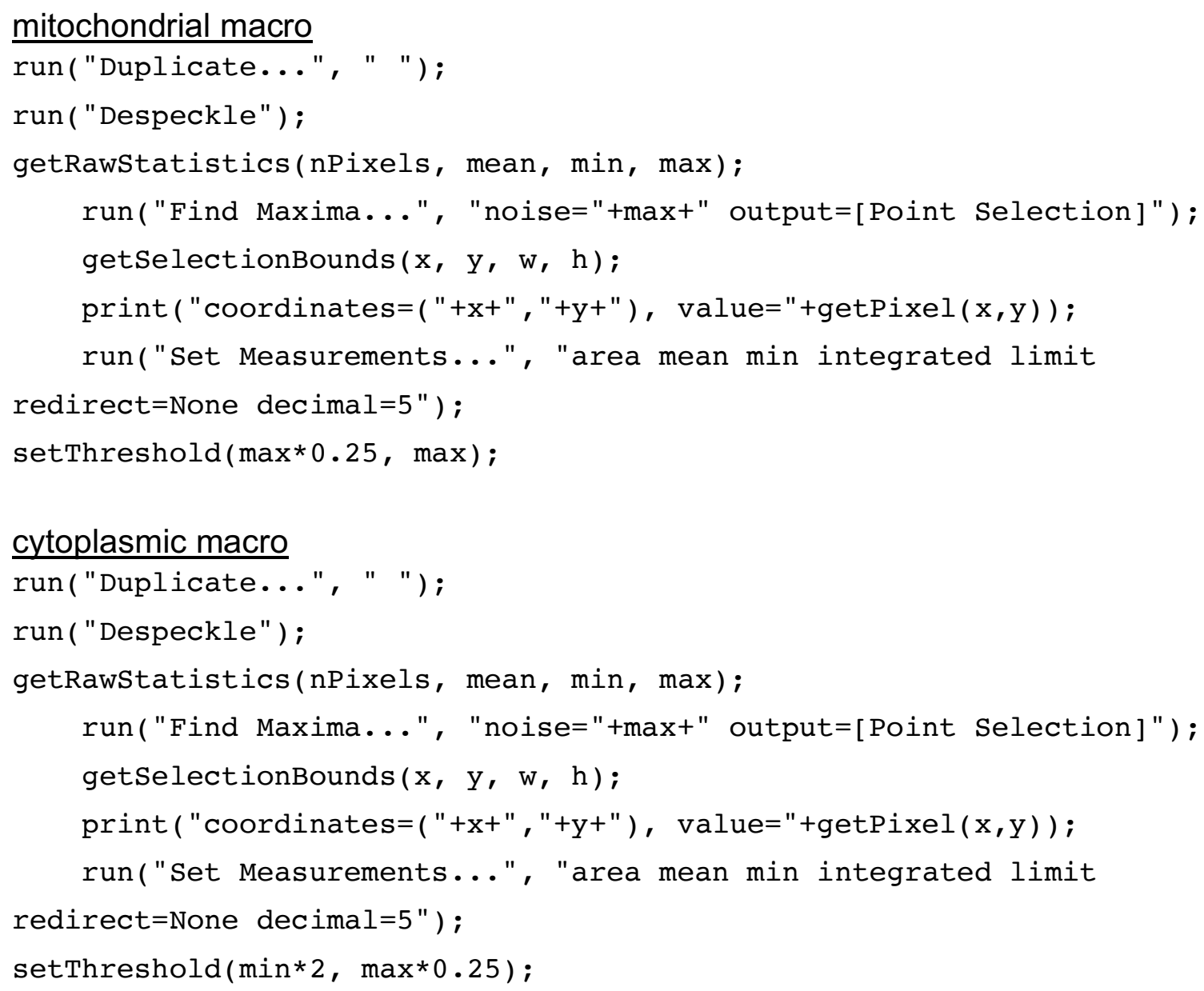




\section{noise macro}

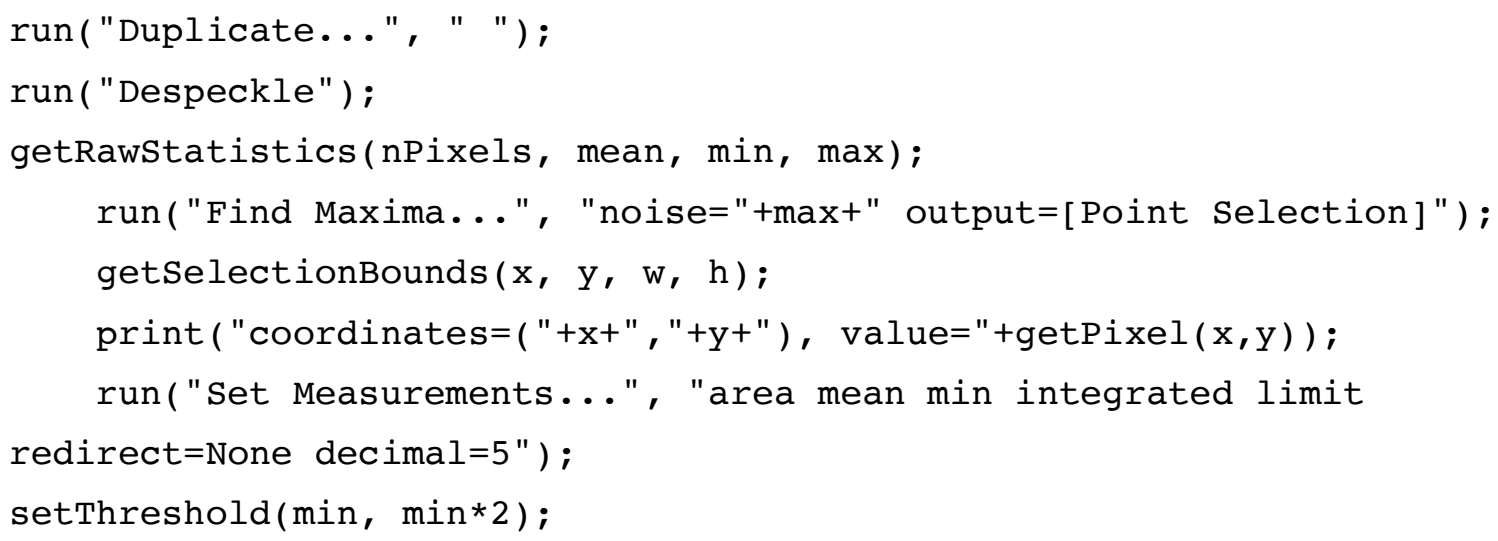

Representative example of compartmentalized images is displayed in Figure S12. The intensities for each compartment were obtained from these measurements.
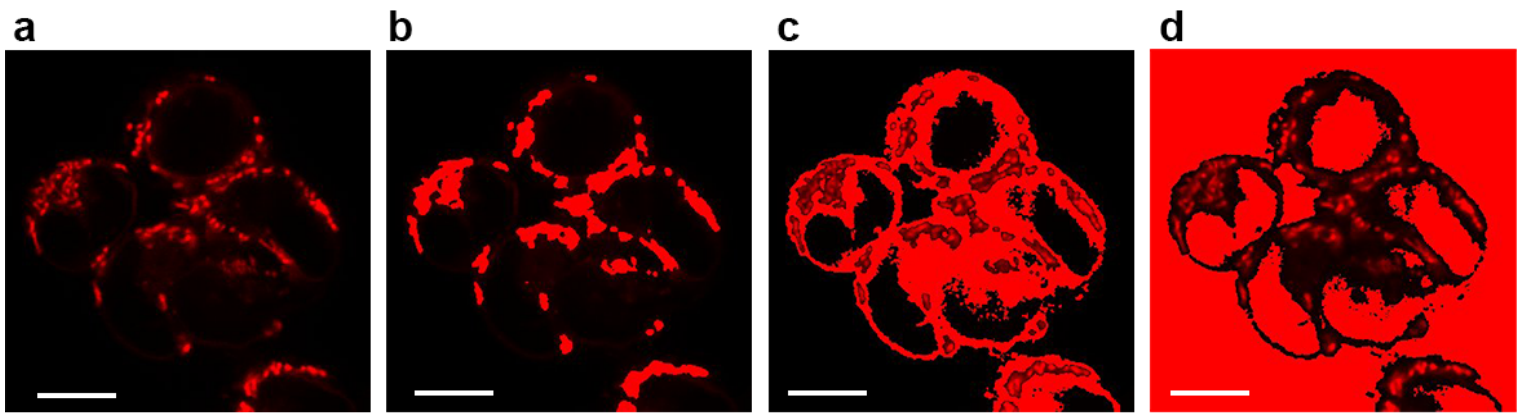

Figure S12. a, Example of red image of mitochondria. HEK293 cells were treated with probe $5(1 \mu \mathrm{M})$ for $2 \mathrm{~h}$. b. Image of mitochondria fraction (red) obtained employing "mitochondrial macro" on image a. c, Image of cytoplasm (red) obtained employing "cytoplasm macro" on image a. d, Image of noise fraction obtained employing "noise macro" on image a. Scale bar $=10 \mu \mathrm{m}$.

\section{Co-localization of LC3 punctae and Mitochondria}

Recruitment of LC3 to autophagosomes and mitochondria was assessed by quantification of the overlay of the signals of LC3-mTurquoise 2 with the signals obtained from activation of either probe 1 or 3 . The cells were counterstained with Hoechst 33342 to identify the nuclei. Good quality images, with strong signal but avoiding saturation, were collected in the blue $\left(\lambda_{\mathrm{ex}}=405 \mathrm{~nm} ; \lambda_{\mathrm{em}}=450 \pm 50 \mathrm{~nm}\right)$, $\operatorname{cyan}\left(\lambda_{\mathrm{ex}}=445 \mathrm{~nm} \mathrm{~nm} ; \lambda_{\mathrm{em}}=470 \pm 24 \mathrm{~nm}\right)$, and $\operatorname{red}\left(\lambda_{\mathrm{ex}}=561 \mathrm{~nm} ; \lambda_{\mathrm{em}}=630 \pm 75 \mathrm{~nm}\right)$ imaging channels. The images were collected using a 100x objective (100x 1.49 CFI 
Apo TIRF), an EMCCD camera (Digital Orca Flash 4.0 V2, rolling shutter usage, $2048 \times 2048$ pixel, $6.5 \times 6.5 \mu \mathrm{m})$ with no binning $(1 \times 1)$. Representative images are shown in Figure $S 13$.

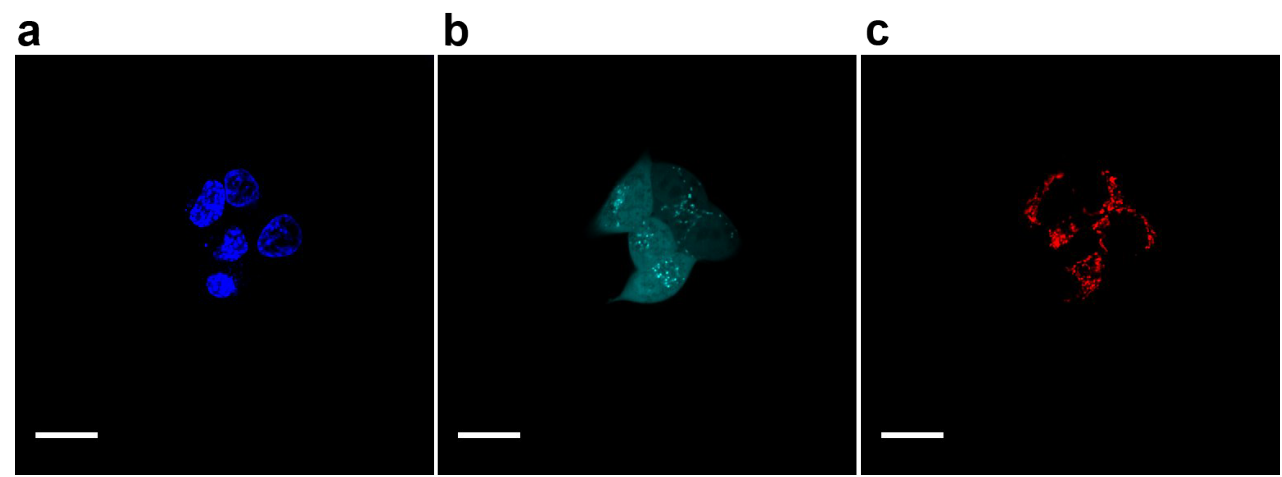

Figure S13. Representative images of a, Nuclei (blue channel); b, LC3-mTurquiose2 (cyan channel), and c, Mitochondria (red channel). Scale bar $=5 \mu \mathrm{m}$.

Binary images were generated by segmentation of the cyan and red images. For the cyan images, the following macro was applied in Fiji (ImageJ):

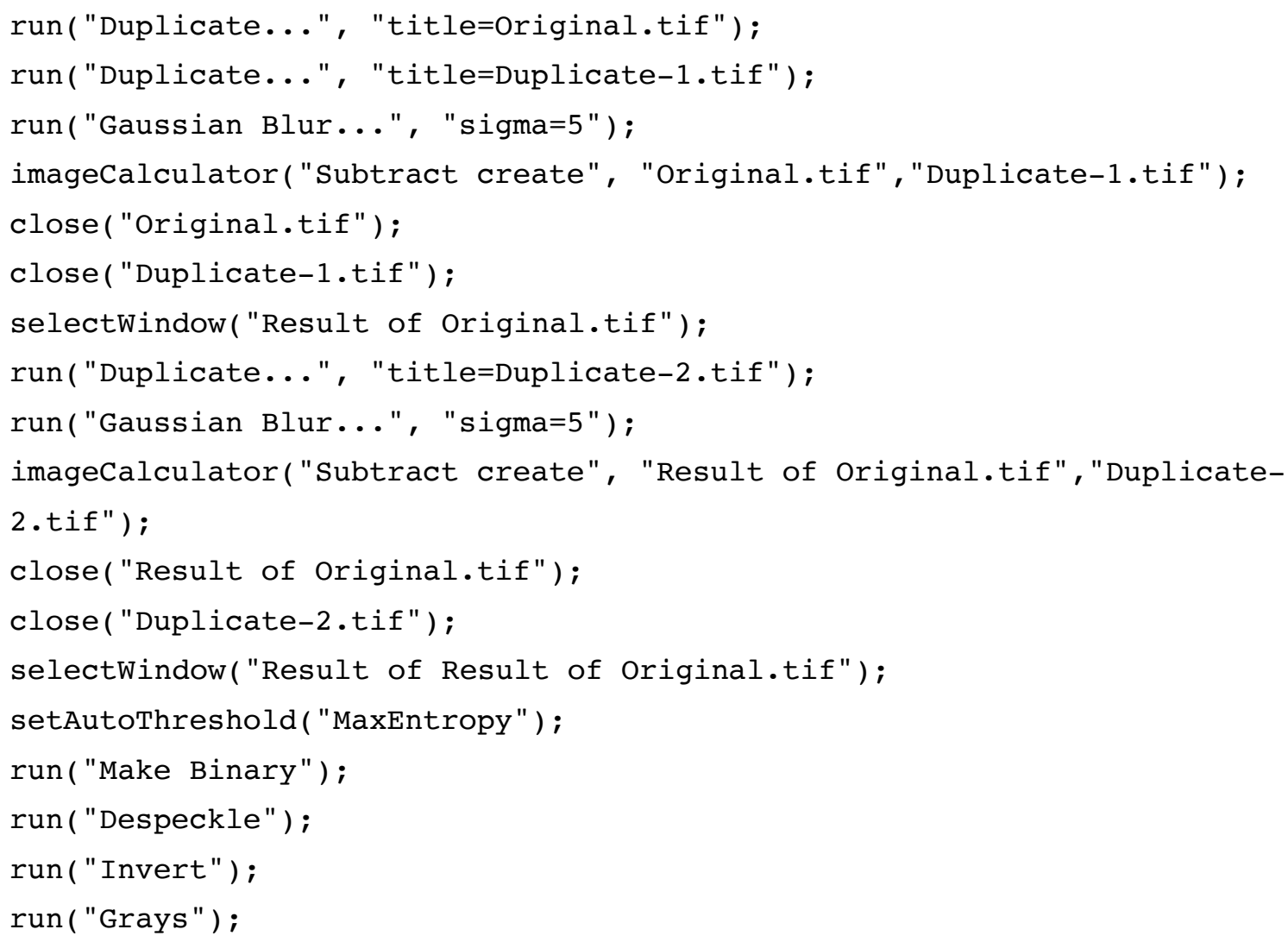


For the red images, the following macro was applied:

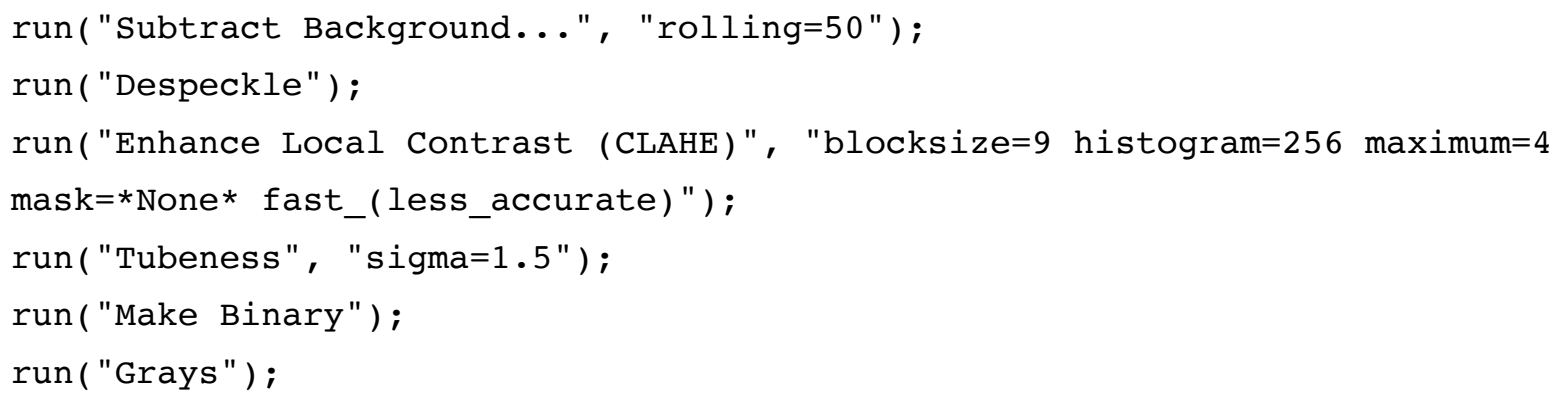

Application of these macros generated binary images of LC3 vesicles and mitochondria. Representative examples are displayed in Figure S14.
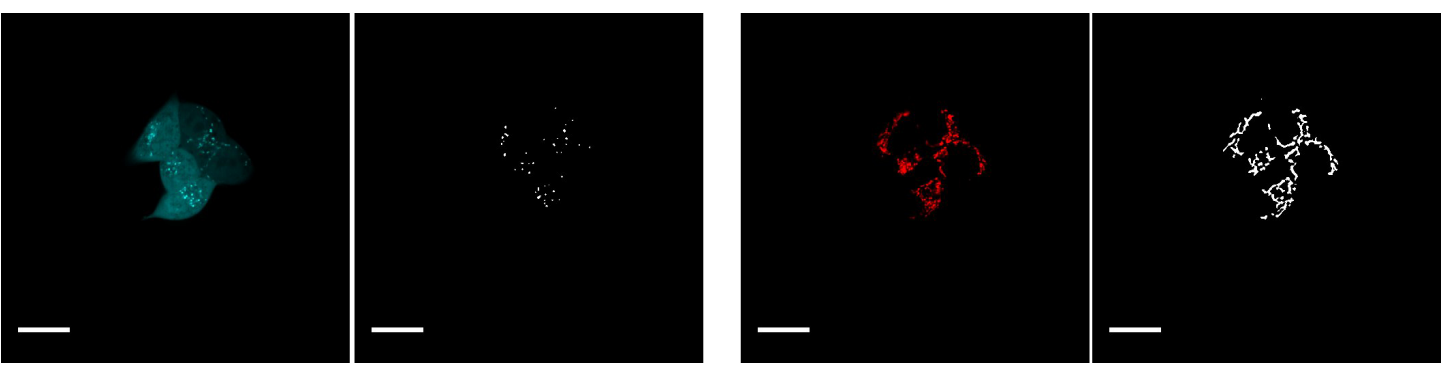

Figure S14. Examples of cyan and red images and their segmented, binary images obtained employing the macros described above. Scale bar $=5 \mu \mathrm{m}$.

The LC3 vesicles that co-localize with mitochondria were isolated by combining the two binary images in Fiji with the "Image calculator" and the "AND" function (Figure S15).
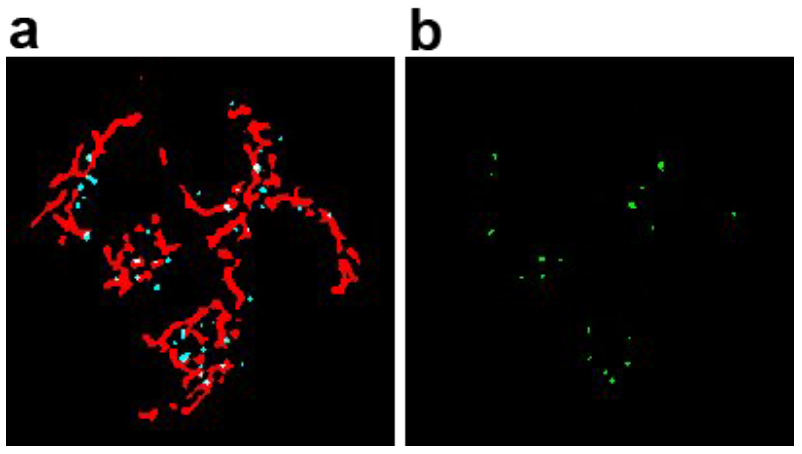

Figure S15. a, Overlay of mitochondria (red) and vesicles (cyan) binary images. b, Extracted vesicles that overlay with mitochondria using the AND function in Fiji. 
The number of co-localized vesicles and mitochondria per cell was obtained using CellProfiler 3.0.0 (cellprofiler.org). Individual cells were identified as secondary objects of nuclei, which were identified as primary objects from raw blue channel images (Figure S16a-b). Cytosols were identified as secondary objects employing the identified nuclei and the image from the cyan channel, followed by masking to remove the area of the nuclei (Figure S16c-d). Finally, the extracted vesicles from the binary images (Figure S16b) were related to the identified cytosols and counted (Figure S16e). The CellProfiler pipeline file is available upon request.

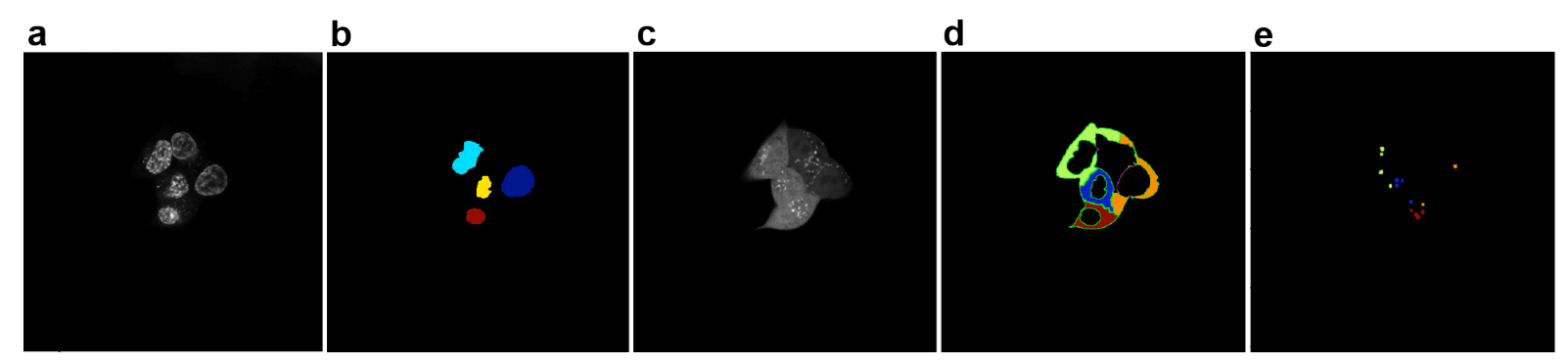

Figure S16. a, Raw image of nuclei. b, Segmentation of image (a) and identification of nuclei. c, Raw image of the cyan channel containing vesicles and cytosolic signals. d, Identification of cytosols as secondary objects based on the cyan image and the identified nuclei in image (b). e, Identification of overlaid vesicles and mitochondria per cell. 


\section{Probe Characterization}

\section{Synthesis of Probes}

\section{1,2,3,3-Tetramethyl-3H-indol-1-ium iodide (5)}

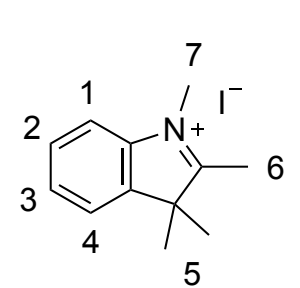

2,3,3-Trimethyl-3H-indole $2(1.0 \mathrm{~mL}, 6.3 \mathrm{mmol})$ and iodomethane $(0.5 \mathrm{~mL}, 7.5 \mathrm{mmol})$ were combined in a microwave vial and then sealed. The reaction mixture was heated at $120{ }^{\circ} \mathrm{C}$ in the microwave, set at very high absorbance for $20 \mathrm{~min}$. The solid formed was washed with $\mathrm{Et}_{2} \mathrm{O}$ to afford $\mathbf{5}$ as a pale yellow solid $(1.26 \mathrm{~g}, 4.20 \mathrm{mmol}$, 67\%). ${ }^{1} \mathrm{H}$ NMR (400 MHz, DMSO-d6) $\delta=7.91$ (dd, $J=8.6,2.4 \mathrm{~Hz}, 1 \mathrm{H}, \mathrm{H} 1$ ), 7.82 (dd, $J=8.6,2.4 \mathrm{~Hz}, 1 \mathrm{H}, \mathrm{H} 4), 7.66-7.59(\mathrm{~m}, 2 \mathrm{H}, \mathrm{H} 2 / 3), 3.97$ (s, 3H, H7), 2.76 (s, $3 \mathrm{H}, \mathrm{H} 6), 1.52(\mathrm{~s}, 6 \mathrm{H}, \mathrm{H} 5) \mathrm{ppm} .{ }^{13} \mathrm{C}$ NMR $(101 \mathrm{MHz}, \mathrm{DMSO}-\mathrm{d} 6) \delta=196.0,142.1$, 141.6, 129.3, 128.8, 123.3, 115.1, 53.9, 34.6, 21.7, 14.0 ppm. HRMS (ESI): exact mass calculated for $\left[\mathrm{C}_{12} \mathrm{H}_{16} \mathrm{~N}\right]^{+}:$: 174.1277; found: 174.1276 .

\section{(E)-1,3,3-Trimethyl-2-(4-nitrostyryl)-3H-indol-1-ium iodide (3)}

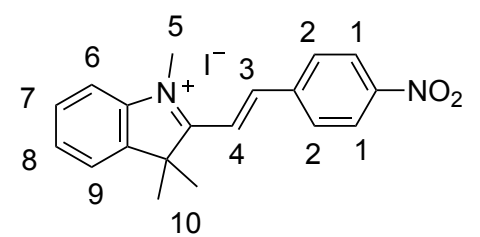

1,2,3,3-Tetramethyl-3H-indol-1-ium iodide $\mathbf{5}$ (50 mg, $0.17 \mathrm{mmol}$ ) and 4-nitrobenzaldehyde (25 $\mathrm{mg}, 0.17 \mathrm{mmol})$ were dissolved in $\mathrm{EtOH}(5 \mathrm{~mL})$ and triethylamine $(50 \mu \mathrm{L})$ was added to the solution. The reaction mixture was stirred at reflux for $18 \mathrm{~h}$. The solvent was evaporated, the residue was dissolved in a minimum amount of $\mathrm{CH}_{2} \mathrm{Cl}_{2}$ and precipitated from $\mathrm{Et}_{2} \mathrm{O}$ to afford 3 (27 mg, $0.06 \mathrm{mmol}, 37 \%)$ as a red solid. $\mathrm{R}_{\mathrm{f}}\left(\mathrm{SiO}_{2}, \mathrm{CH}_{2} \mathrm{Cl}_{2} / \mathrm{MeOH} 9: 1\right)=0.52 .{ }^{1} \mathrm{H}$ NMR $(400$ $\left.\mathrm{MHz}, \mathrm{CD}_{3} \mathrm{CN}\right) \delta=8.41-8.35(\mathrm{~m}, 2 \mathrm{H}, \mathrm{H} 2), 8.27(\mathrm{~d}, J=16.6 \mathrm{~Hz}, 1 \mathrm{H}, \mathrm{H} 3), 8.22-8.17$ (m, 2H, H1), $7.80-7.74$ (m, 2H, H6/7), $7.73-7.64$ (m, 2H, H8/9), 7.57 (d, J = 16.6 $\mathrm{Hz}, 1 \mathrm{H}, \mathrm{H} 4), 4.12$ (s, 3H, H5), 1.80 (s, 6H, H10) ppm. ${ }^{13} \mathrm{C}$ NMR (101 MHz, CD 3 CN) $\delta=150.8,140.8,131.7,131.6,130.4,125.2,123.9,117.5,116.5,47.5,36.1,25.5$, 9.0 ppm. HRMS (ESI) calculated for $\left[\mathrm{C}_{19} \mathrm{H}_{19} \mathrm{~N}_{2} \mathrm{O}_{2}\right]^{+}: 307.1441$, found 307.1445 . 
(Z)-Tributyl(1-(4-nitrophenyl)-2-(1,3,3-trimethylindolin-2-

ylidene)ethyl)phosphonium iodide (1)

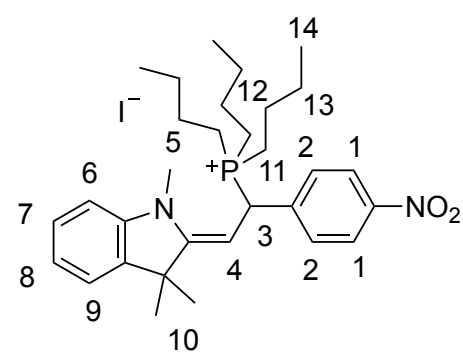

Compound 3 (13 mg, $0.03 \mathrm{mmol}$ ) was dissolved in dry $\mathrm{CH}_{2} \mathrm{Cl}_{2}(0.5 \mathrm{~mL})$ in a Schlenk flask under inert atmosphere at room temperature. $n$-Tributyl phosphine $(15 \mu \mathrm{L}, 0.06 \mathrm{mmol})$ in $\mathrm{CH}_{2} \mathrm{Cl}_{2}(0.1 \mathrm{~mL})$ was added dropwise to the stirring solution and an instant color change from orange to pale pink was observed. The crude product was purified by flash column chromatography $\left(\mathrm{SiO}_{2} ; \mathrm{CH}_{2} \mathrm{Cl}_{2}\right.$ to $\mathrm{CH}_{2} \mathrm{Cl}_{2} / \mathrm{CH}_{3} \mathrm{OH}$ 98:2) to afford the nitro phosphonium 1 (17 mg, $0.026 \mathrm{mmol}, 89 \%$ ) as a yellow solid. ${ }^{1} \mathrm{H}$ NMR $\left(300 \mathrm{MHz}, \mathrm{CD}_{3} \mathrm{CN}\right) \delta=8.27(\mathrm{dd}, J=8.2,6.6 \mathrm{~Hz}, 2 \mathrm{H}, \mathrm{H} 2)$, 7.81 (dt, $J=8.6,2.9 \mathrm{~Hz}, 2 \mathrm{H}, \mathrm{H} 1$ ), $7.19-7.08(\mathrm{~m}, 2 \mathrm{H}), 6.83-6.64(\mathrm{~m}, 2 \mathrm{H}), 5.04$ (dd, $J=16.8,11.9 \mathrm{~Hz}, 1 \mathrm{H}), 4.64(\mathrm{t}, J=11.5 \mathrm{~Hz}, 1 \mathrm{H}), 3.17(\mathrm{~s}, 3 \mathrm{H}), 2.28-2.17\left(\mathrm{~m}, \mathrm{PBu}_{3}\right)$, 1.63 (s, 3H, H10), 1.44 (dt, J = 6.3, $\left.3.5 \mathrm{~Hz}, \mathrm{PBu}_{3}\right), 1.17$ (s, 3H, H10), 0.90 (t, J = $\left.7.2 \mathrm{~Hz}, \mathrm{PBu}_{3}\right)$ ppm. ${ }^{31} \mathrm{P} \mathrm{NMR}\left(122 \mathrm{MHz}, \mathrm{CD}_{3} \mathrm{CN}\right) \delta=36.9,35.8 \mathrm{ppm}$.

\section{(E)-2-(4-Aminostyryl)-1,3,3-trimethyl-3H-indol-1-ium iodide (2)}

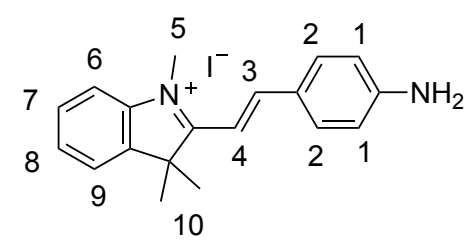

1,2,3,3-Tetramethyl-3H-indol-1-ium iodide 5 (136 mg, $0.54 \mathrm{mmol}$ ) and 4-aminobenzaldehyde (65 mg, $0.54 \mathrm{mmol})$ were dissolved in $\mathrm{EtOH}(13 \mathrm{~mL})$ and the reaction mixture was stirred at reflux for $5 \mathrm{~h}$. The crude

product was purified by flash column chromatography $\left(\mathrm{SiO}_{2} ; \mathrm{CH}_{2} \mathrm{Cl}_{2}\right.$ to $\mathrm{CH}_{2} \mathrm{Cl}_{2} / \mathrm{CH}_{3} \mathrm{OH}$ 9:1) to afford 2 (148 $\left.\mathrm{mg}, 0.37 \mathrm{mmol}, 81 \%\right)$ as a red solid. $\mathrm{R}_{\mathrm{f}}\left(\mathrm{SiO}_{2}\right.$, $\left.\mathrm{CH}_{2} \mathrm{Cl}_{2} / \mathrm{MeOH}: 9 / 1\right)=0.22 .{ }^{1} \mathrm{H} \mathrm{NMR}\left(400 \mathrm{MHz}, \mathrm{CD}_{3} \mathrm{OD}\right) \delta=8.27(\mathrm{~d}, J=15.6 \mathrm{~Hz}, 1 \mathrm{H}$, $\mathrm{H} 3$ ), 7.84 (d, J = 8.7 Hz, 2H, H2), $7.67-7.49$ (m, 4H, H6/7/8/9), 7.17 (d, J = $15.6 \mathrm{~Hz}$, $1 \mathrm{H}, \mathrm{H} 4), 6.76(\mathrm{~d}, J=8.9 \mathrm{~Hz}, 2 \mathrm{H}, \mathrm{H} 1), 3.95$ (s, 3H, H5), 1.80 (s, 6H, H10) ppm. HRMS (ESI) calculated for $\left[\mathrm{C}_{19} \mathrm{H}_{21} \mathrm{~N}_{2}\right]^{+}: 277.1699$, found 277.1701 . 


\section{NMR Spectra}

${ }^{1} \mathrm{H}$ NMR (400 MHz, DMSO-d $)$ spectrum of 5

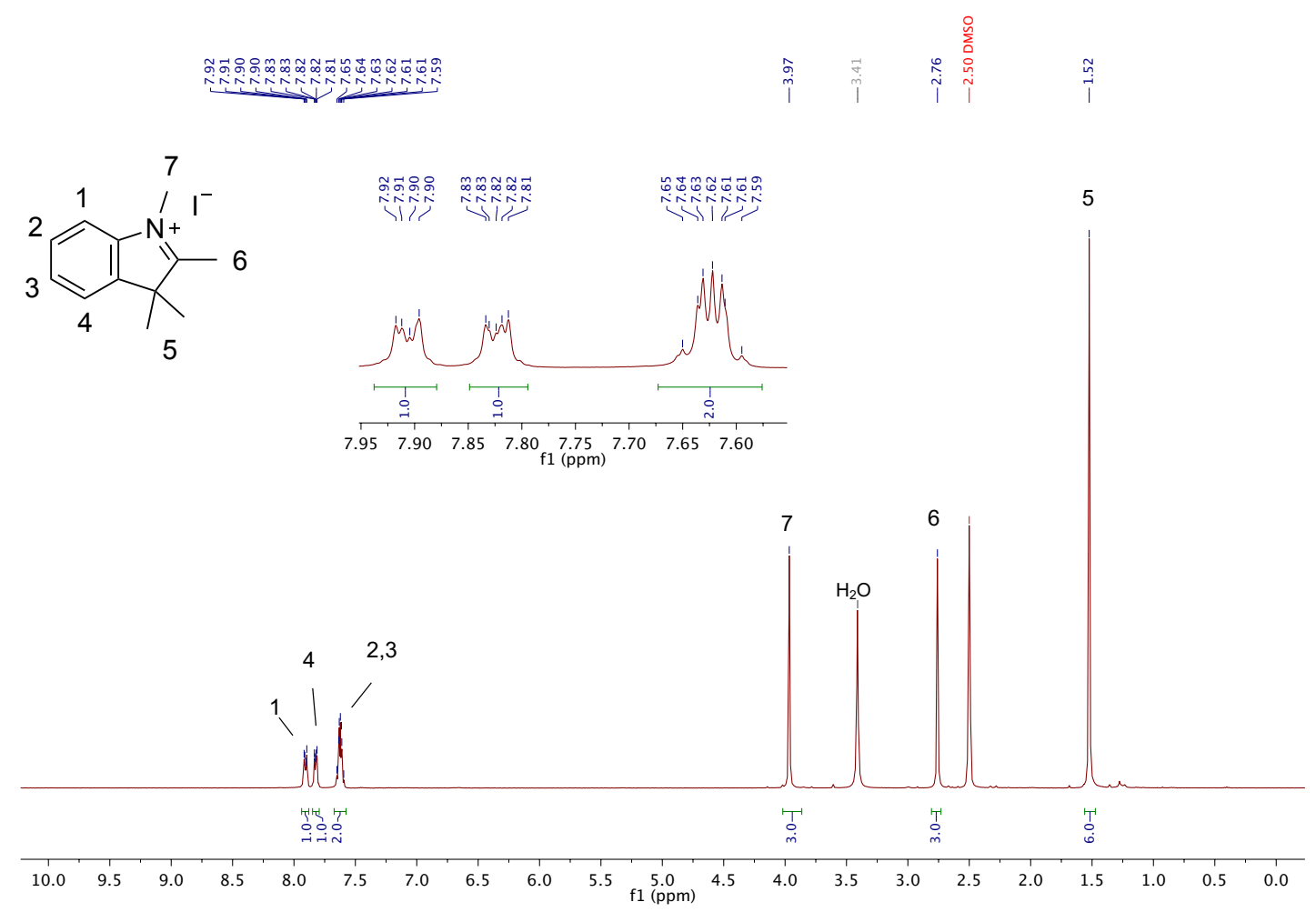

${ }^{13} \mathrm{C}$ NMR $\left(101 \mathrm{MHz}, \mathrm{DMSO}-d_{6}\right)$ spectrum of 5

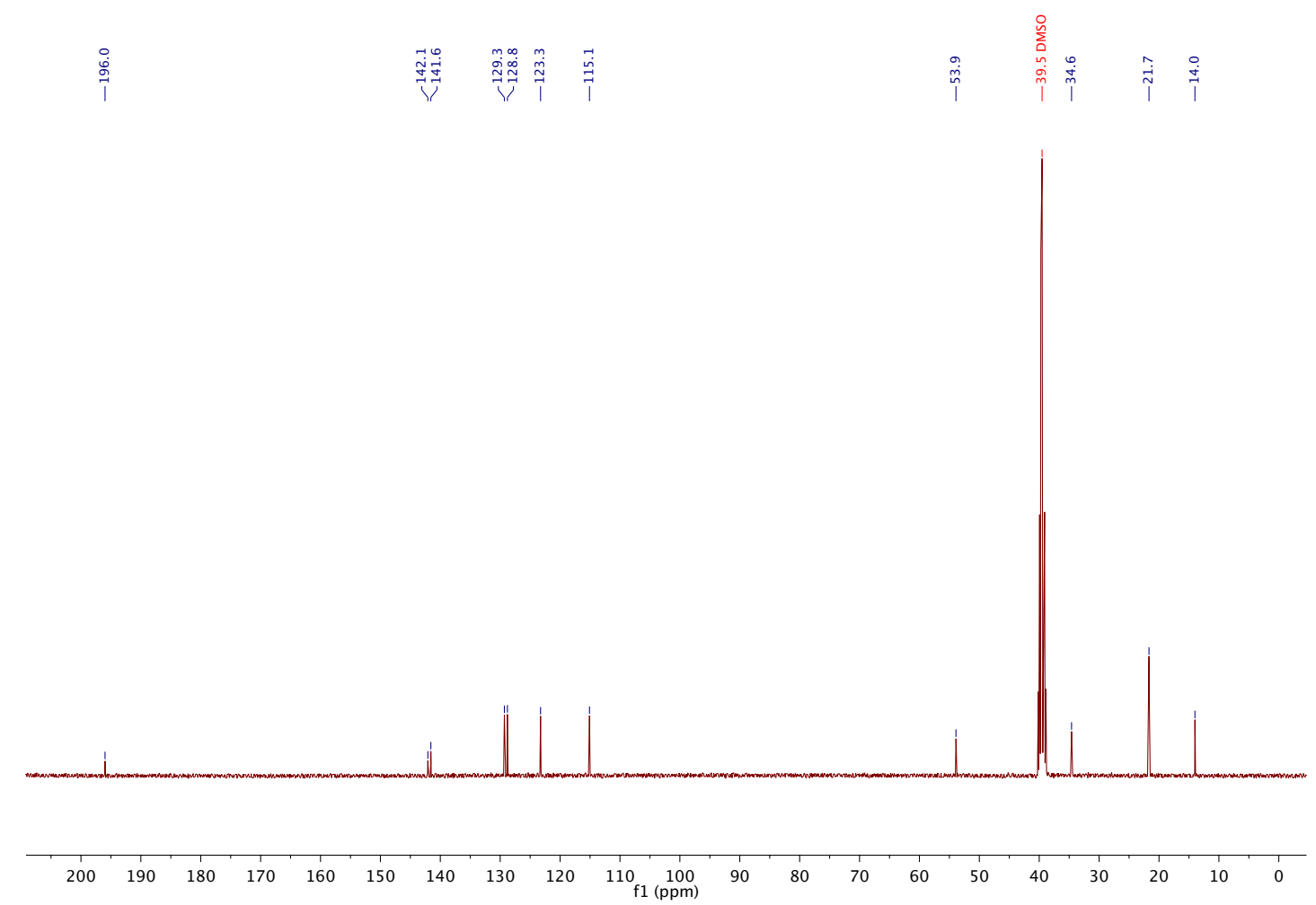

${ }^{1} \mathrm{H}$ NMR $\left(400 \mathrm{MHz}, \mathrm{CD}_{3} \mathrm{CN}-d_{3}\right)$ spectrum of 3 


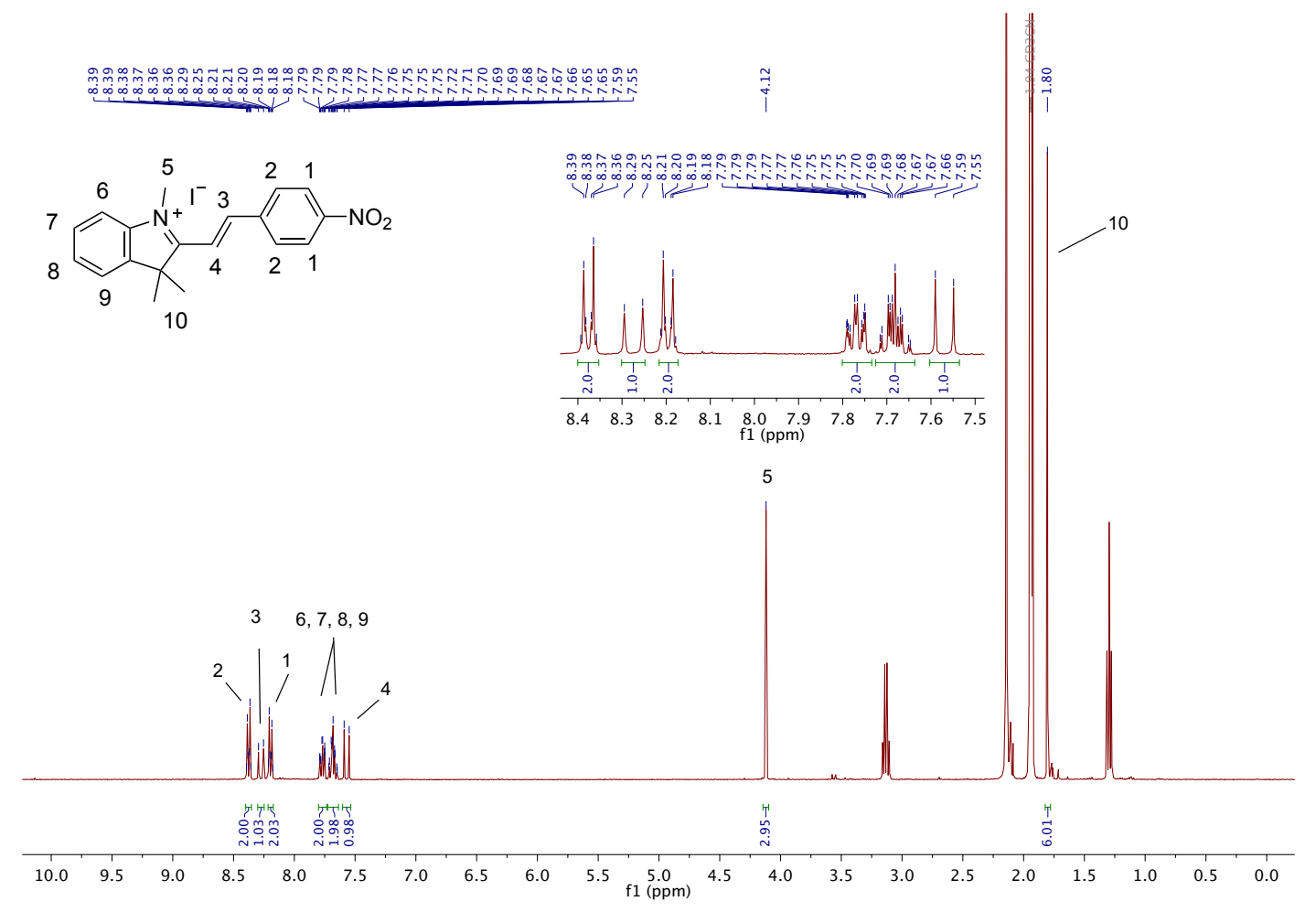

${ }^{13} \mathrm{C}$ NMR $\left(101 \mathrm{MHz}, \mathrm{CD}_{3} \mathrm{CN}-d_{3}\right)$ spectrum of 3

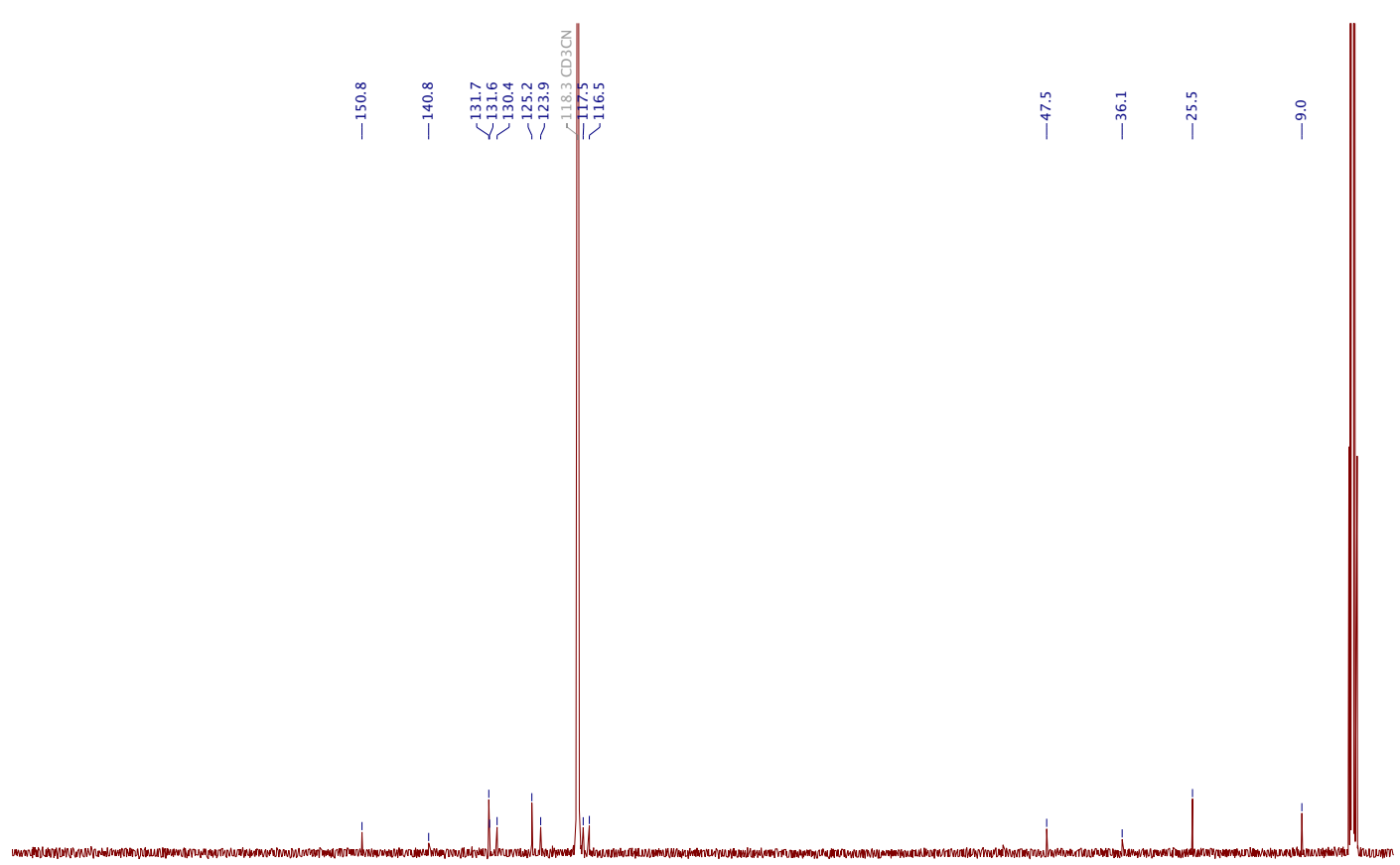

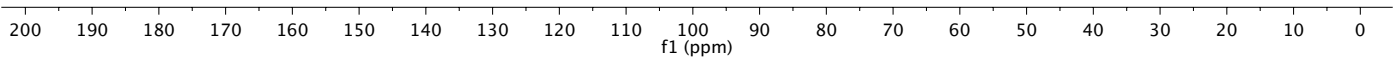


${ }^{1} \mathrm{H}$ NMR $\left(300 \mathrm{MHz}, \mathrm{CD}_{3} \mathrm{CN}-d_{3}\right)$ spectrum of 1

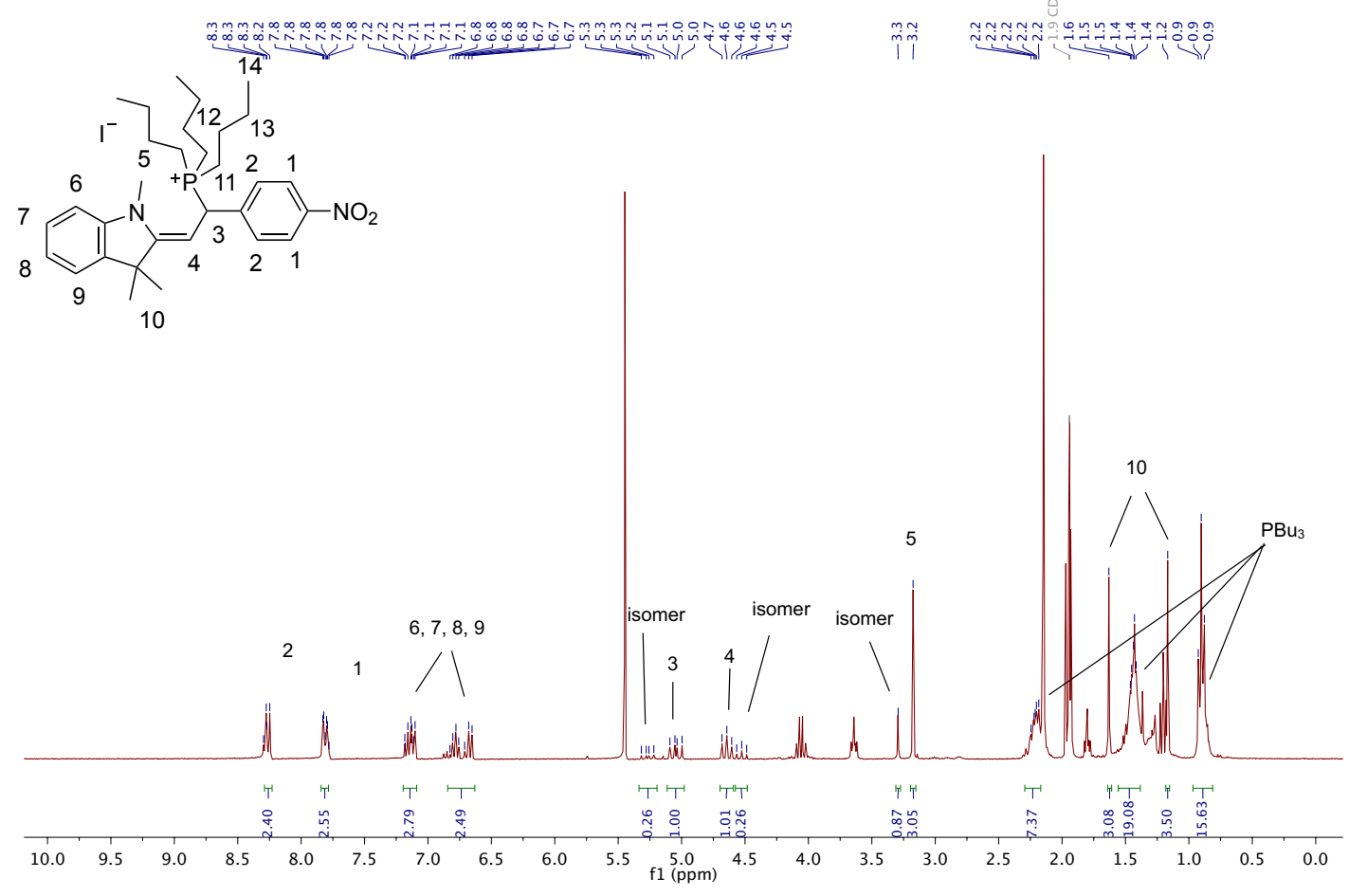

${ }^{31} \mathrm{P}$ NMR (122 MHz, $\left.\mathrm{CD}_{3} \mathrm{CN}-d_{3}\right)$ spectrum of 1 
${ }^{1} \mathrm{H}$ NMR $\left(400 \mathrm{MHz}, \mathrm{CD}_{3} \mathrm{OD}-d_{4}\right)$ spectrum of 2

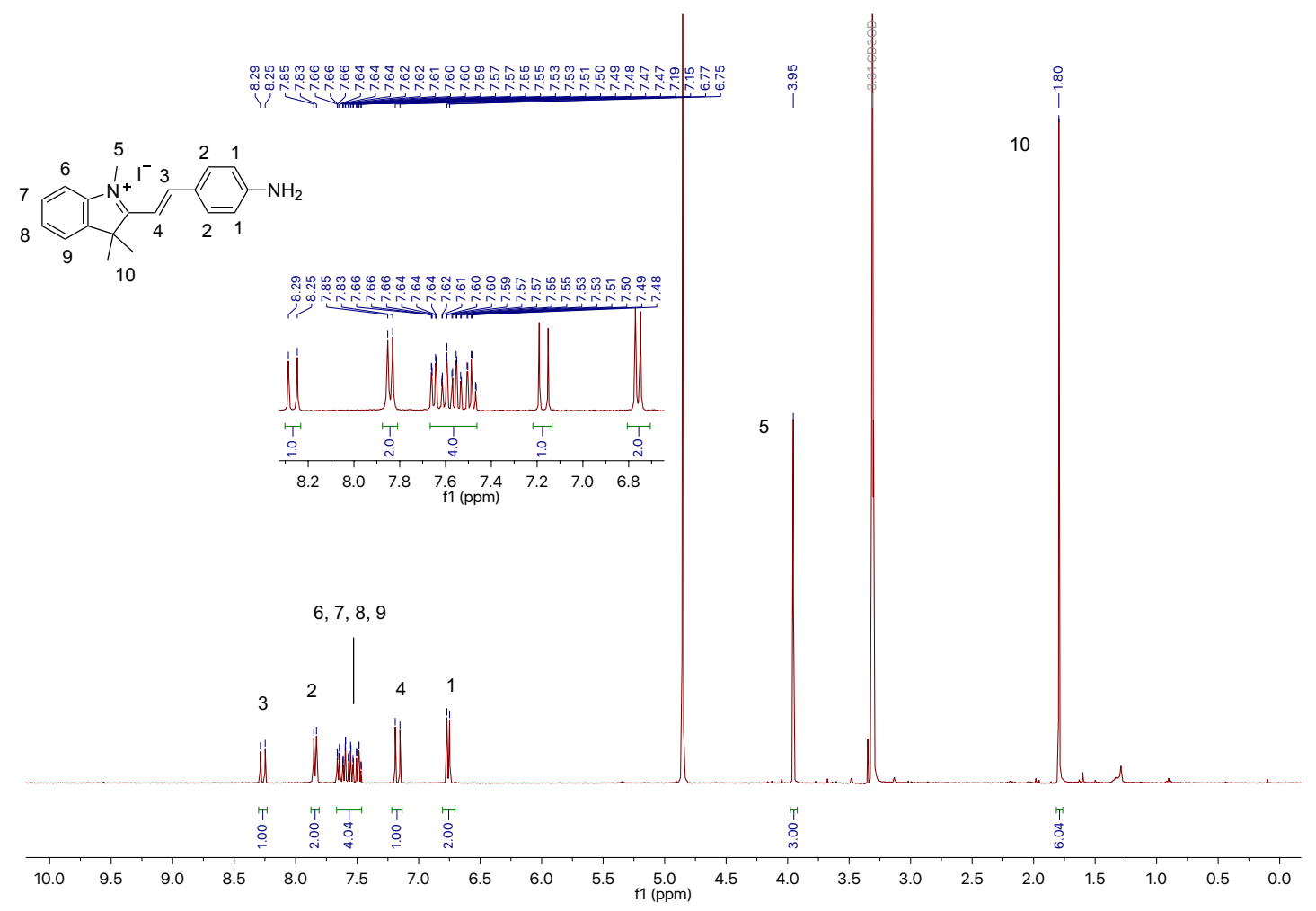

${ }^{13} \mathrm{C}$ NMR $\left(101 \mathrm{MHz}, \mathrm{CD}_{3} \mathrm{OD}-d_{4}\right)$ spectrum of 2

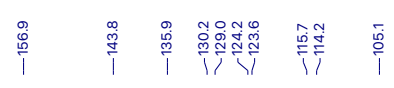

||c:

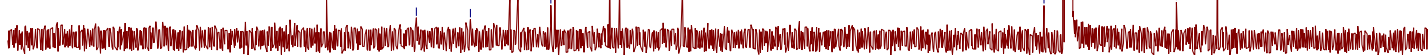

$\begin{array}{lllllllllllllllllllllllll}1 & 100 & 190 & 180 & 170 & 160 & 150 & 140 & 130 & 120 & 110 & \begin{array}{c}100 \\ \mathrm{f1}(\mathrm{ppm})\end{array} & 90 & 80 & 70 & 60 & 50 & 40 & 30 & 20 & 10 & 0\end{array}$ 